\title{
THE LIKELIHOOD RATIO TEST FOR THE NUMBER OF COMPONENTS IN A MIXTURE WITH MARKOV REGIME
}

\author{
Elisabeth Gassiat ${ }^{1}$ And Christine Keribin $^{1}$
}

\begin{abstract}
We study the LRT statistic for testing a single population i.i.d. model against a mixture of two populations with Markov regime. We prove that the LRT statistic converges to infinity in probability as the number of observations tends to infinity. This is a consequence of a convergence result of the LRT statistic for a subproblem where the parameters are restricted to a subset of the whole parameter set.

Résumé. Nous étudions la statistique du test de rapport de vraisemblance (TRV) pour tester un modèle i.i.d. à une population contre un mélange de deux populations à régime markovien. Nous prouvons que la statistique du TRV converge vers l'infini en probabilité quand la taille de l'échantillon tend vers l'infini. Ceci est une conséquence d'un résultat de convergence de la statistique du TRV pour un sous-problème où les paramètres sont restreints à un sous-ensemble de l'ensemble complet des paramètres.
\end{abstract}

AMS Subject Classification. 62F05, 62H30, 62A10.

Received June 7, 1999. Revised April 20, 2000.

\section{INTRODUCTION}

Hidden Markov Models have been used for a long time as a useful modeling tool. Applications include speech processing (Rabiner [32]), neurophysiology (Fredkin and Rice [17]), biology (Churchill [6]), see also Mac Donald and Zucchini [27]. A hidden Markov model (HMM) is a discrete time stochastic process $\left(Y_{k}\right)_{k \in \mathbb{N}}$ such that the distribution of the process may be well explained through a non observable Markov chain $\left(X_{k}\right)_{k \in \mathbb{N}}$ taking values in a finite state space. Given $\left(X_{k}\right),\left(Y_{k}\right)$ is a sequence of independent random variables, and the distribution of $Y_{n}$ given $\left(X_{k}\right)$ is that of $Y_{n}$ given $X_{n}$. Thus, the one dimensional marginal of the process, namely the distribution of $Y_{n}$, appears to be a mixture of populations where the number of populations $m$ is the cardinality of the state space of the hidden Markov chain. The observations may then be said to follow a "mixture model with Markov regime".

Asymptotic properties of maximum likelihood estimators (m.l.e.) for the estimation of the parameters in a HMM have received early interest when the $\left(Y_{k}\right)$ take values in a finite set (Baum and Petrie [2]), and recent interest in the general case (Leroux [24], Ryden [33], Bickel and Ritov [3], Bickel et al. [4], Mevel [29], Vandekerkhove [34], Douc and Matias [13], Jensen and Petersen [21]). Various algorithms have been proposed to achieve the m.l.e., see for instance Mevel [29] or Vandekerkhove [34].

Keywords and phrases: Likelihood ratio test, hidden Markov model, order of a mixture.

1 Laboratoire Modélisation Stochastique et Statistique, Université d'Orsay, bâtiment 425, 91405 Orsay, France;

e-mail: Elisabeth.Gassiat@math.u-psud.fr

(C) EDP Sciences, SMAI 2000 
The problem of estimating $m$, the number of populations, or of testing between different values of $m$, is an important one either for numerical features (a larger value than the true one leads to flat likelihoods) or for its interpretation as a meaningful parameter. Earlier works on the subject propose to use likelihood methods (Leroux [24], Ryden [33], Finesso [16]), but fail to prove the consistency of the estimator (or the asymptotic level of the test) because of an intrinsic difficulty of the model: the usual regularity conditions are not fulfilled under the null hypothesis, so that the $\chi$-square theory may not apply. Corresponding problem for mixtures with i.i.d. regime has a long history (see Lindsay [26]) but the asymptotic distribution of the LRT (likelihood ratio test) has been recently solved by Dacunha-Castelle and Gassiat [10,11] and the consistency of the penalized likelihood estimator has been proved by Keribin [23].

The aim of this paper is the study of the LRT for testing the number of populations in a mixture with Markov regime. Intuition and methods of Dacunha-Castelle and Gassiat [10,11] may be applied, and it appears that the computation of the asymptotic distribution in the general setting is much more complicated than in the i.i.d. regime situation. However, by applying the method to a subproblem of the general problem, we obtain meaningful results. Let us now be more precise.

Let

$$
\mathcal{F}=\left\{f_{\gamma}, \gamma \in \Gamma\right\}
$$

be a set of probability densities with respect to a given measure $\nu$ on a metric space, where $\Gamma$ is a compact subset of some Euclidean space. Let $\left(X_{k}\right)_{k \in \mathbb{N}}$ be a stationary Markov chain taking values in a set of $m$ distinct values of $\Gamma,\left\{\gamma_{1}, \ldots, \gamma_{m}\right\}$, with transition matrix $\Pi$. The observations are $\left(Y_{1}, \ldots, Y_{n}\right)$. Conditionally to $\left(X_{1}, \ldots, X_{n}\right)$, $\left(Y_{1}, \ldots, Y_{n}\right)$ are independent variables, each $Y_{i}$ having conditional distribution $f_{X_{i}} \cdot \nu$. Define

$$
T_{n}(m)=\sup \left\{\ell_{n}\left(\Pi, \gamma_{1}, \ldots, \gamma_{m} ; m\right): \Pi m \times m \text { stochastic matrix, }\left(\gamma_{1}, \ldots, \gamma_{m}\right) \in \Gamma^{m}\right\},
$$

in which $\ell_{n}\left(\Pi, \gamma_{1}, \ldots, \gamma_{m} ; m\right)$ is the log-likelihood of the observations under the parameter $\left(\Pi, \gamma_{1}, \ldots, \gamma_{m}\right)$ when the number of hidden states is $m$. One of our main results is that the LRT statistic $T_{n}(2)-T_{n}(1)$ for testing i.i.d. observations of one population against 2 populations with stationary Markov regime converges in probability to infinity under the null hypothesis of i.i.d. observations (see Th. 2.5). This result is a consequence of another convergence result in the sub-problem of testing a fixed contamination with stationary Markov regime. Let $f_{\gamma_{0}}$ and $f_{\gamma_{1}}$ be fixed densities in $\mathcal{F}, \gamma_{0} \neq \gamma_{1}$. Let $\left(X_{k}\right)_{k \in \mathbb{N}}$ take values in $\left\{\gamma_{0}, \gamma_{1}\right\}$, with initial distribution

$$
P\left(X_{0}=\gamma_{0}\right)=1-u, \quad P\left(X_{0}=\gamma_{1}\right)=u
$$

and transition matrix

$$
\Pi_{u, q}=\left(\begin{array}{cc}
1-\frac{q u}{1-u} & \frac{q u}{1-u} \\
q & 1-q
\end{array}\right) .
$$

Here, $0 \leq q \leq 1,0 \leq u \leq 1$, and $q$ and $u$ are also constrained by

$$
0 \leq \frac{q u}{1-u} \leq 1
$$

This is a general parametrization of the distribution of a stationary Markov chain with two parameters. We want to test the null hypothesis $H_{0}$ : "the $Y_{i}$ 's are i.i.d. with common density $f_{\gamma_{0}}$ ", against the alternative $H_{1}$ : " $\left(Y_{n}\right)_{n \in \mathbb{N}}$ follows a HMM for some parameters $u, q$ ", and with fixed $\gamma_{0}$ and $\gamma_{1}$. Obviously, the HMM reduces to i.i.d. variables with common density $f_{\gamma_{0}}$ if and only if $u=0$, so that the test we consider is that of $H_{0}$ : "u=0"

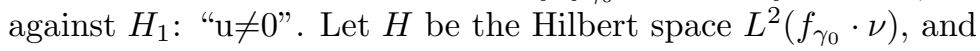

$$
N^{2}=\left\|\frac{f_{\gamma_{1}}-f_{\gamma_{0}}}{f_{\gamma_{0}}}\right\|_{H}^{2} .
$$


For any $\eta \in] 0,1[$, let

$$
\mathcal{Q}_{\eta}=\left\{q \in[0,1]:(1-q)^{2}\left(N^{2}+1\right) \leq 1-\eta\right\}=\left[q_{\eta}, 1\right]
$$

with

$$
q_{\eta}=1-\sqrt{\frac{1-\eta}{N^{2}+1}}>0 .
$$

When the $q$ 's are restricted to belong to $\mathcal{Q}_{\eta}$, let $\mathcal{P}_{\eta}$ be the parameter set of possible $(u, q)$. The log-likelihood $\ell_{n}\left(\Pi_{u, q}, \gamma_{0}, \gamma_{1} ; 2\right)$ will now be denoted by $\ell_{n}(u, q)$. Notice that under the null hypothesis, the parameter $q$ is undefined, the log-likelihood does not depend on $q$, and is $\ell_{n}(0, q)$ for any $q$. The LRT statistic is now, when the $q$ 's are restricted to belong to $\mathcal{Q}_{\eta}$ :

$$
V_{n}(\eta)=\sup \left\{\ell_{n}(u, q)-\ell_{n}(0, q):(u, q) \in \mathcal{P}_{\eta}\right\} .
$$

The first main convergence result is that $V_{n}(\eta)$ converges to half the supremum of the square of the positive part of some Gaussian process (see Th. 2.1). This result is similar to that obtained for testing the number of populations in a mixture, or for testing the order of a stationary ARMA process, see Dacunha-Castelle and Gassiat $[10,11]$. As $\eta$ goes to 0 , the covariance of infinitely many members of the process goes to 0 , and the convergence to infinity of $T_{n}(2)-T_{n}(1)$ is thus an easy consequence.

Convergence to infinity of LRT's has been observed earlier in two papers about mixtures with i.i.d. regime: when the parameter set is unbounded, see Hartigan [19], and when the family $\mathcal{F}$ is not regular, two situations in which the set of scores may be proved to be non compact, see Ciuperca [7]. Here, the parameter set is compact, and the family $\mathcal{F}$ is regular. Our result may be compared to a similar result, not proved in any paper but which is also an easy consequence of Theorem 4.5 in Dacunha-Castelle and Gassiat [11], that the LRT for testing the order of an ARMA model tends to infinity when the poles and roots are not restricted to a compact subset of the open unit complex disk.

Our result leaves open the problem of the choice of the penalization rate when estimating the number of populations using penalized likelihood estimators, for mixtures with Markov regime. However, for translation or scale mixtures, the method proposed by Dacunha-Castelle and Gassiat [9] may be applied to mixtures with Markov regime to obtain a consistent estimate of the number of populations.

The paper is organized as follows. In Section 2, we state the assumptions and our main results. Section 3 is devoted to numerical experiments, and Section 4 to the proofs of intermediate results.

\section{MAin RESUlts}

We study the asymptotic properties of the LRT under the null hypothesis, in which the $Y_{i}$ 's are assumed to be i.i.d. with distribution $f_{\gamma_{0}} \nu$. Let us introduce the assumptions:

- (A1) For $\gamma \in\left\{\gamma_{0}, \gamma_{1}\right\}$, under $H_{0}, E\left(\left|\log f_{\gamma}\left(Y_{1}\right)\right|\right)<+\infty$.

- (A2) Under $H_{0}$, the support of the random variable $\frac{f_{\gamma_{1}}}{f_{\gamma_{0}}}\left(Y_{1}\right)$ is $[0,+\infty[$.

- (A3) Under $H_{0}, E\left(\frac{f_{\gamma_{0}}}{f_{\gamma_{1}}}\left(Y_{1}\right)\right)<+\infty$.

- (A4) Under $H_{0}, E\left(\frac{f_{\gamma_{1}}}{f_{\gamma_{0}}}\left(Y_{1}\right)\right)^{3}<+\infty$.

Let now $\left(\xi_{q}\right)_{q \in \mathcal{Q}_{\eta}}$ be the continuous Gaussian process with covariance

$$
R\left(q_{1}, q_{2}\right)=\frac{C\left(q_{1}, q_{2}\right)}{C\left(q_{1}, q_{1}\right)^{1 / 2} C\left(q_{2}, q_{2}\right)^{1 / 2}}
$$


with

$$
C\left(q_{1}, q_{2}\right)=\frac{1-\left(1-q_{1}\right)\left(1-q_{2}\right)}{1-\left(1-q_{1}\right)\left(1-q_{2}\right)\left(N^{2}+1\right)} .
$$

Then:

Theorem 2.1. Assume that (A1, A2, A3) and (A4) hold. Under $H_{0}, V_{n}(\eta)$ converges in distribution to

$$
\frac{1}{2} \sup _{q \in \mathcal{Q}_{\eta}} \xi_{q}^{2} \cdot 1_{\xi_{q} \geq 0}
$$

Proof of Theorem 2.1. Theorem 2.1 will be proved by investigating the asymptotic behavior of the maximum of the likelihood for fixed $q$ as a process with $\mathcal{Q}_{\eta}$ the time set, and then taking the maximum for $q$ over $\mathcal{Q}_{\eta}$. For an HMM with parameters $\Pi_{u, q}, \gamma_{0}$ and $\gamma_{1}$, let $\left(p_{k}(u, q)\right)_{k \geq 1}$ be the prediction filter, that is, for any positive $k$ :

$$
p_{k}(u, q)=P\left(X_{k}=\gamma_{1} / Y_{1}, \ldots, Y_{k-1}\right) .
$$

It is well known that the $p_{k}(u, q)$ 's satisfy the recursive equation:

$$
p_{k+1}(u, q)=\frac{\frac{q u}{(1-u)}\left(1-p_{k}\right) f_{\gamma_{0}}\left(Y_{k}\right)+(1-q) p_{k} f_{\gamma_{1}}\left(Y_{k}\right)}{\left(1-p_{k}\right) f_{\gamma_{0}}\left(Y_{k}\right)+p_{k} f_{\gamma_{1}}\left(Y_{k}\right)}
$$

with initialization $p_{1}(u, q)=u$. Parameter $(u, q)$ is omitted for $p_{k}$ on the right hand of the recurrence equation for readability. This will be the case also in further recurrence equations concerning the derivatives of the prediction filter. Now the log-likelihood may be written:

$$
\ell_{n}(u, q)-\ell_{n}(0, q)=\sum_{k=1}^{n} \log \left[1+p_{k}(u, q) \frac{f_{\gamma_{1}}-f_{\gamma_{0}}}{f_{\gamma_{0}}}\left(Y_{k}\right)\right] .
$$

Define

$$
S_{n}(u, q)=\frac{1}{n}\left[\ell_{n}(u, q)-\ell_{n}(0, q)\right] .
$$

Asymptotic properties of $S_{n}(u, q)$ and its derivatives will be investigated using the properties of the Markov chains $\left(Y_{k}, p_{k}(u, q)\right)_{k \in \mathbb{N}}\left(Y_{k} \text {, derivatives of } p_{k}(u, q)\right)_{k \in \mathbb{N}}$. This point of view is the one adopted by Mevel [29], Mevel and LeGland [30] to obtain the asymptotic properties of the maximum likelihood estimator (m.l.e.) of the parameters when the number of populations $m$ is known.

Let now $(\widehat{u}, \widehat{q})$ be the m.l.e. for $(u, q)$, and $\widehat{u}_{q}$ the m.l.e. for $u$ when $q$ is fixed. We have:

Proposition 2.2. Assume (A1, A2, A3) and (A4). Then $\sup _{q \in \mathcal{Q}_{\eta}} \widehat{u}_{q}$ and $\widehat{u}$ converge to 0 in probability under $H_{0}$.

Remark. This result could be derived from Theorem 3 of Leroux [24]. However, we give here an alternative specific proof which shows the effect of overestimating $m$.

The limiting contrast function of $S_{n}(u, q)$ will be proved to be

$$
S(u, q)=-\int K\left(f_{\gamma_{0}},(1-p) f_{\gamma_{0}}+p f_{\gamma_{1}}\right) d \mu_{u, q}(p),
$$

in which $K(\cdot, \cdot)$ is the Kullbak information (see for instance Dacunha-Castelle and Duflo [8] for a definition) and $\mu_{u, q}$ is a probability distribution on $[0,1]$ such that, for $u=0, \mu_{0, q}$ is the dirac at 0 . Notice that $S(u, q)$ is always finite by (A1), and, since the Kullback information is always non negative, for any $(u, q), S(u, q) \leq 0$, and 
$S(0, q)=0$. Proposition 2.2 will follow from the fact that $S(u, q)=0$ only for $u=0$, and that the convergence of $S_{n}(u, q)$ to $S(u, q)$ is uniform enough, see Section 4 . We have:

$$
V_{n}(\eta)=\sup _{q \in \mathcal{Q}_{\eta}} n S_{n}\left(\hat{u}_{q}, q\right)
$$

But using Proposition 2.2, for any $q$ in $\mathcal{Q}_{\eta}$, Taylor expansion leads to

$$
S_{n}\left(\widehat{u}_{q}, q\right)=-\frac{1}{2} \frac{\left(\frac{\partial}{\partial u} S_{n}(0, q)\right)^{2}}{\frac{\partial^{2}}{\partial u^{2}} S_{n}(0, q)} \cdot\left(1+R_{n}(q)\right)
$$

in which $R_{n}(q)$ converges in probability to 0 uniformly in $q$ as soon as

$$
\frac{\frac{\partial^{2}}{\partial u^{2}} S_{n}(\tilde{u}, q)}{\frac{\partial^{2}}{\partial u^{2}} S_{n}(0, q)}
$$

converges to 1 uniformly in $q$ for any $\tilde{u}$ converging in probability to 0 uniformly in $q$. Now,

$$
\begin{aligned}
\frac{\partial^{2}}{\partial u^{2}} S_{n}(u, q)= & -\frac{1}{n} \sum_{i=1}^{n} \frac{1}{\left(1+p_{k}(u, q) \frac{f_{\gamma_{1}}-f_{\gamma_{0}}}{f_{\gamma_{0}}}\left(Y_{k}\right)\right)^{2}}\left(\frac{\partial p_{k}}{\partial u}(u, q)\right)^{2}\left(\frac{f_{\gamma_{1}}-f_{\gamma_{0}}}{f_{\gamma_{0}}}\left(Y_{k}\right)\right)^{2} \\
& +\frac{1}{n} \sum_{i=1}^{n} \frac{1}{1+p_{k}(u, q) \frac{f_{\gamma_{1}}-f_{\gamma_{0}}}{f_{\gamma_{0}}}\left(Y_{k}\right)} \frac{\partial^{2} p_{k}}{\partial u^{2}}(u, q) \frac{f_{\gamma_{1}}-f_{\gamma_{0}}}{f_{\gamma_{0}}}\left(Y_{k}\right) .
\end{aligned}
$$

In particular, for $u=0$, one obtains

$$
\frac{\partial^{2}}{\partial u^{2}} S_{n}(0, q)=-\frac{1}{n} \sum_{i=1}^{n}\left(\frac{\partial p_{k}}{\partial u}(0, q)\right)^{2}\left(\frac{f_{\gamma_{1}}-f_{\gamma_{0}}}{f_{\gamma_{0}}}\left(Y_{k}\right)\right)^{2}+\frac{1}{n} \sum_{i=1}^{n} \frac{\partial^{2} p_{k}}{\partial u^{2}}(0, q) \frac{f_{\gamma_{1}}-f_{\gamma_{0}}}{f_{\gamma_{0}}}\left(Y_{k}\right) .
$$

In this sum, the second term is centered, so that as soon as laws of large numbers apply, the limiting value is $-N^{2}$ times the expectation of $\left(\frac{\partial p_{k}}{\partial u}(0, q)\right)^{2}$ under the stationary distribution. This convergence result holds more generally and we have:

Proposition 2.3. Assume that (A2, A3) and (A4) hold. Then, for any random $\tilde{u}$ converging in probability to 0 uniformly in $q$,

$$
\frac{\partial^{2}}{\partial u^{2}} S_{n}(\tilde{u}, q)
$$

converges in probability to $-N^{2} C(q, q)$ uniformly over $\mathcal{Q}_{\eta}$.

Proposition 2.3 leads to

$$
V_{n}(\eta)=\frac{1}{2}\left[\sup _{q \in \mathcal{Q}_{\eta}} \frac{\left(\sqrt{n} \frac{\partial}{\partial u} S_{n}(0, q)\right)^{2}}{N^{2} C(q, q)}\right]\left(1+o_{P}(1)\right)
$$

and Theorem 2.1 will follow from:

Proposition 2.4. Assume (A1, A2) and (A3). Then, the process

$$
\sqrt{n}\left(\frac{\partial}{\partial u} S_{n}(0, q)\right)_{q \in \mathcal{Q}_{\eta}}
$$

converges uniformly to the continuous Gaussian process with covariance $N^{2} C(\cdot, \cdot)$. 
Comments. The score process at $u=0$ and for fixed $q$ is:

$$
\frac{\partial}{\partial u} S_{n}(0, q)=\frac{1}{n} \sum_{k=1}^{n} \frac{\partial p_{k}}{\partial u}(0, q) \frac{f_{\gamma_{1}}-f_{\gamma_{0}}}{f_{\gamma_{0}}}\left(Y_{k}\right)
$$

in which $\left(\frac{\partial p_{k}}{\partial u}(0, q)\right)_{k \in \mathbb{N}}$ is a Markov chain satisfying the recursion:

$$
\frac{\partial p_{k+1}}{\partial u}(0, q)=q+(1-q) \frac{f_{\gamma_{1}}}{f_{\gamma_{0}}}\left(Y_{k}\right) \frac{\partial p_{k}}{\partial u}(0, q)
$$

with initialization $\frac{\partial p_{1}}{\partial u}(0, q)=1$. But in case an invariant distribution exists and has a second moment $\sigma^{2},(15)$ leads to:

$$
\sigma^{2}=q^{2}+2 q(1-q)+(1-q)^{2}\left(N^{2}+1\right) \sigma^{2} .
$$

Since for any $q, q^{2}+2 q(1-q) \geq 0$, this equation leads to a non negative $\sigma^{2}$ if and only if $1-(1-q)^{2}\left(N^{2}+1\right) \geq 0$. This last condition is thus a necessary one to obtain a convergence in distribution of $\sqrt{n} \frac{\partial}{\partial u} S_{n}(0, q)$ and a law of large numbers for $\frac{\partial^{2}}{\partial u^{2}} S_{n}(0, q)$. This is why the parameter set is cut in two different regions, the union of all $\mathcal{P}_{\eta}$, and its complementary set. Our conjecture is that, on this complementary set, $n S_{n}\left(\hat{u}_{q}, q\right)$ converges to half the square of a standard Gaussian white noise.

To state the unboundedness of the LRT statistic for testing between one or two populations in a mixture with Markov regime, we introduce the following assumption:

- (I) $T_{n}(1)-\ell_{n}(0, q)$ is uniformly tight under $H_{0}$.

Notice that a sufficient condition for $(\mathrm{I})$ to hold is that the family $\mathcal{F}$ is regular enough. For instance, differentiability in quadratic mean with respect to $\gamma$ and existence and inversibility of the Fisher information suffice since in this case $T_{n}(1)-\ell_{n}(0, q)$ converges to a $\chi^{2}(1)$ (see for instance Van der Vaart [35]). We now obtain the unconstrained result:

Theorem 2.5. Assume (I), and that (A1, A2, A3) and (A4) hold for any $\gamma_{0}$ and $\gamma_{1}$ in $\Gamma$. Then, under $H_{0}$, $T_{n}(2)-T_{n}(1)$ converges to infinity in probability.

Comments. When testing the number of parameters of a model, different situations occur. We consider the test with as alternative more parameters than the null hypothesis $H_{0}$. When the set of parametric distributions is smooth enough and for compact sets of parameters, the LRT statistic converges in general in distribution under $H_{0}$. In case all parameters are identifiable when the model is overparametrized, the LRT statistic is asymptotically chi-square (see [22]). In case some parameters are not identifiable under $H_{0}$, as for mixtures, the asymptotic distribution is no longer chi-square but still exists. This allows to set tests with asymptotic known level. This allows also to use compensated likelihood for the estimation of the number of parameters [23], with a compensator of form $-v_{n} \times n b$ of parameters, where $v_{n} \rightarrow+\infty$ and $v_{n}=o(n)$.

Here the LRT statistic tends to infinity. In some sense, this means that the alternative is too far from the null (for some of its points). But this is not due to the non compactness of the set of parameters as in [19], or to the non regularity of the distribution as in [7]. To be able to construct tests with known asymptotic level, one has to know at least the speed of convergence. Indeed, let a test be

$$
1_{T_{n}(2)-T_{n}(1) \geq s_{n}}
$$

where $s_{n} \rightarrow+\infty$. Under the alternative, as usual, using [4], $T_{n}(2)-T_{n}(1)$ is of order $n \times C$, for a constant $C$, so that the power of the test is asymptotically one as soon as $s_{n}=o(n)$. The asymptotic level will be 0 as soon as, under $H_{0}, T_{n}(2)-T_{n}(1)$ converges to $+\infty$ slower than $s_{n}$. The speed of convergence of $T_{n}(2)-T_{n}(1)$ will be investigated in further work. 
Proof of Theorem 2.5. Let $f_{\gamma_{0}} \nu$ be the true (unknown) distribution of the i.i.d. variables $\left(Y_{k}\right)$. Let $\gamma_{1}$ be a point in $\Gamma$ distinct of $\gamma_{0}$.

Since the LRT statistic for i.i.d. observations with the model $\mathcal{F}$ is tight, $T_{n}(2)-T_{n}(1)$ converges to infinity in probability as soon as $V_{n}(0)$ does.

Let $\rho<1$, and define for all integer $k$ :

$$
r_{k}=\rho^{k^{2}}
$$

Define also $q_{k} \in[0,1]$ by

$$
\left(1-q_{k}\right)^{2}\left(N^{2}+1\right)=1-r_{k} .
$$

Obviously,

$$
V_{n}(0) \geq \sup \left\{\ell_{n}\left(\widehat{u_{q_{k}}}, q_{k}\right)-\ell_{n}\left(0, q_{k}\right): k \in \mathbb{N}\right\}
$$

Now, for the particular sequence $r_{k}=\rho^{k^{2}}$, observe that

$$
\lim _{k \neq l \rightarrow+\infty} R\left(q_{k}, q_{l}\right)=0
$$

so that

$$
\sup _{k \leq L} \xi_{q_{k}}^{2} \cdot 1_{\xi_{q_{k}} \geq 0}
$$

converges in probability to $+\infty$ as $L$ tends to $+\infty$. For any positive $M$, for any positive $\epsilon$, there exists $L_{0}$ such that

$$
P\left(\sup _{k \leq L_{0}} \xi_{q_{k}}^{2} \cdot 1_{\xi_{q_{k}} \geq 0}>M\right) \geq 1-\epsilon .
$$

But using portmanteau's lemma, the fact that $\sup \left\{\ell_{n}\left(\widehat{u_{q_{k}}}, q_{k}\right)-\ell_{n}\left(0, q_{k}\right): k \leq L_{0}\right\}$ converges in distribution to $\sup _{k \leq L_{0}} \xi_{q_{k}}^{2} \cdot 1_{\xi_{q_{k}} \geq 0}$, and inequality (16), we have that

$$
\liminf _{n \rightarrow+\infty} P\left(V_{n}(0)>M\right) \geq 1-\epsilon .
$$

Since this holds for any $\epsilon, V_{n}(0)$ tends to $+\infty$ in probability.

\section{Remarks.}

- The same result holds if the parameter space is restricted to all possible $(u, q)$ such that

$$
(1-q)^{2}\left(N^{2}+1\right) \leq 1,
$$

as may be seen from the proof. This means that the unboundedness of the LRT statistic is not due to the fact that the model contains non ergodic hidden Markov chains (by taking $q=0$ ), but to the fact that the alternative may be too far from the null hypothesis, also with respect to the distance between the populations involved in the mixture.

- When the hidden chain has $m$ states and true parameters $\Pi_{0}, \gamma_{1}, \ldots \gamma_{m}$, the statistic $T_{n}(m)-\ell_{n}\left(\Pi_{0}\right.$, $\left.\gamma_{1}, \ldots \gamma_{m}\right)$ converges to a chi-square distribution, as a result of the LAN properties and the $\sqrt{n}$-convergence of the maximum likelihood estimator for HMM's with known number of hidden states [3,4], under appropriate regularity assumptions. We conjecture that the convergence to infinity of $T_{n}(m+q)-T_{n}(m)$ may be proved using the same ideas as in this paper.

- Stationary Markov chains are particular Markov chains, so that the general alternative handling with eventually non stationary chains leads to similar results. 


\section{NumERICAL EXPERIMENTS}

In this section, we numerically investigate the behavior of the LRT statistic. The true model is an i.i.d. process with Gaussian distribution $\mathcal{N}(0,1)$ of mean 0 and variance 1. We test i.i.d. observations of one population with distribution $\mathcal{N}(0,1)$ against two populations with Markov regime, the distributions of the observations conditionally to the Markov chain being $\mathcal{N}(0,1)$ and $\mathcal{N}(1,1)$. In other words $f_{\gamma_{0}}(x)=(2 \pi)^{-1 / 2} \exp -\frac{x^{2}}{2}$ and $f_{\gamma_{1}}(x)=(2 \pi)^{-1 / 2} \exp -\frac{(x-1)^{2}}{2}$.

We first draw the likelihood map, then we study the convergence in distribution of $V_{n}(\eta)$ (Th. 2.1) and we finally experiment the convergence to infinity of the LRT statistic (Th. 2.5).

\subsection{Likelihood map}

We compute the log-likelihood ratio (LLR)

$$
\ell_{n}(u, q)-\ell_{n}(0, q)=\sum_{k=1}^{n} \log \left[1+p_{k}(u, q) \frac{f_{\gamma_{1}}-f_{\gamma_{0}}}{f_{\gamma_{0}}}\left(Y_{k}\right)\right]
$$

using the recursive equation of the prediction filter (8) for $(q, u) \in[0,1] \times[0,1]$, such that $q u /(1-u) \leq 1$, by step of 0.01. The typical aspect of all our LLR maps is the following: we observe the LLR to fast decrease with $u$, and refine the map for $u$ near 0 . Two cases happen:

- Case A: the maximum is 0 , reached for $\hat{u}=0$, and the LLR is an always decreasing function of $u$ (Fig. 1);

- Case B: the maximum is strictly positive and is reached for $\hat{u}$ close to 0 (Fig. 2).

When the number of observations increases, $\hat{u}$ gets closer to 0 . For 10000 observations, $\hat{u}$ is of the order of 0.01 , for 50000 observations, $\hat{u}$ is of the order of 0.005 , and for 100000 observations, $\hat{u}$ is of the order of 0.001 .

As the LLR can take large negative values, we cut off the values larger than -3 and replaced them by -3 to allow the visualization of the maximum. In the same way, we represent the points of the domain where $q u /(1-u) \geq 1$ with the value -4 .
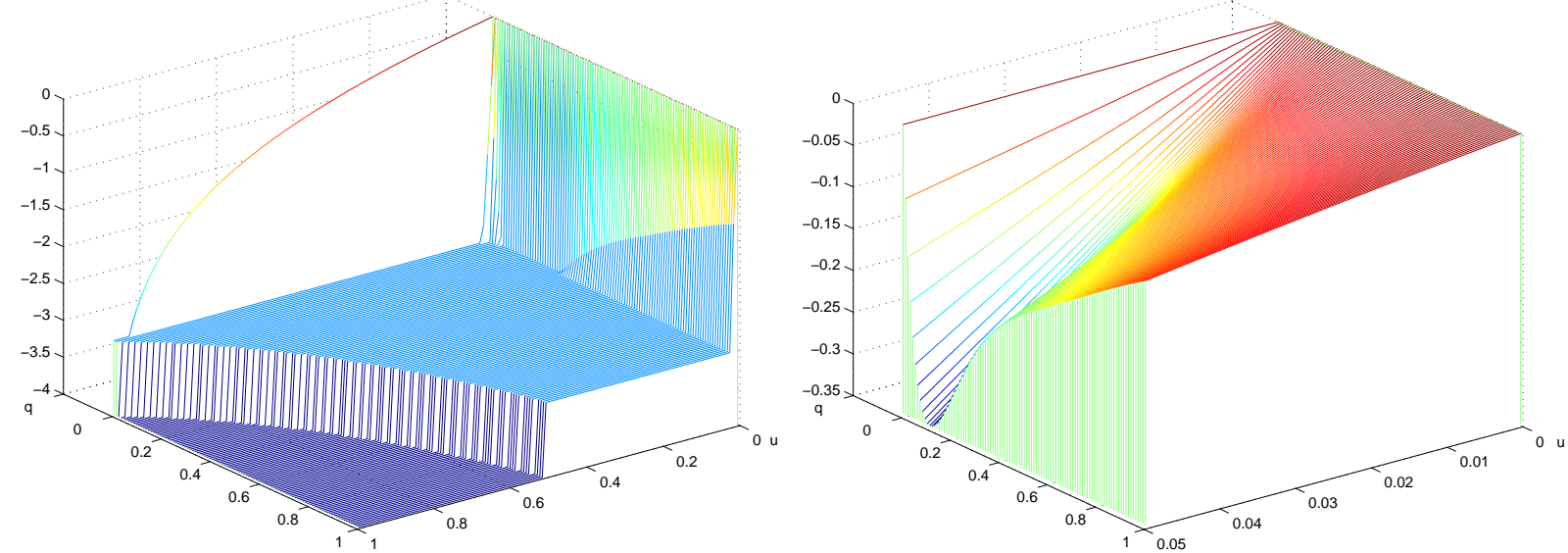

Figure 1. Case A. On left: on the whole domain $(q, u) \in[0,1] \times[0,1]$ by steps of 0.01 . On right: refinement $(q, u) \in[0,1] \times[0,0.05]$ by steps of $5.10^{-4}$ for $u$ and 0.01 for $q$. 

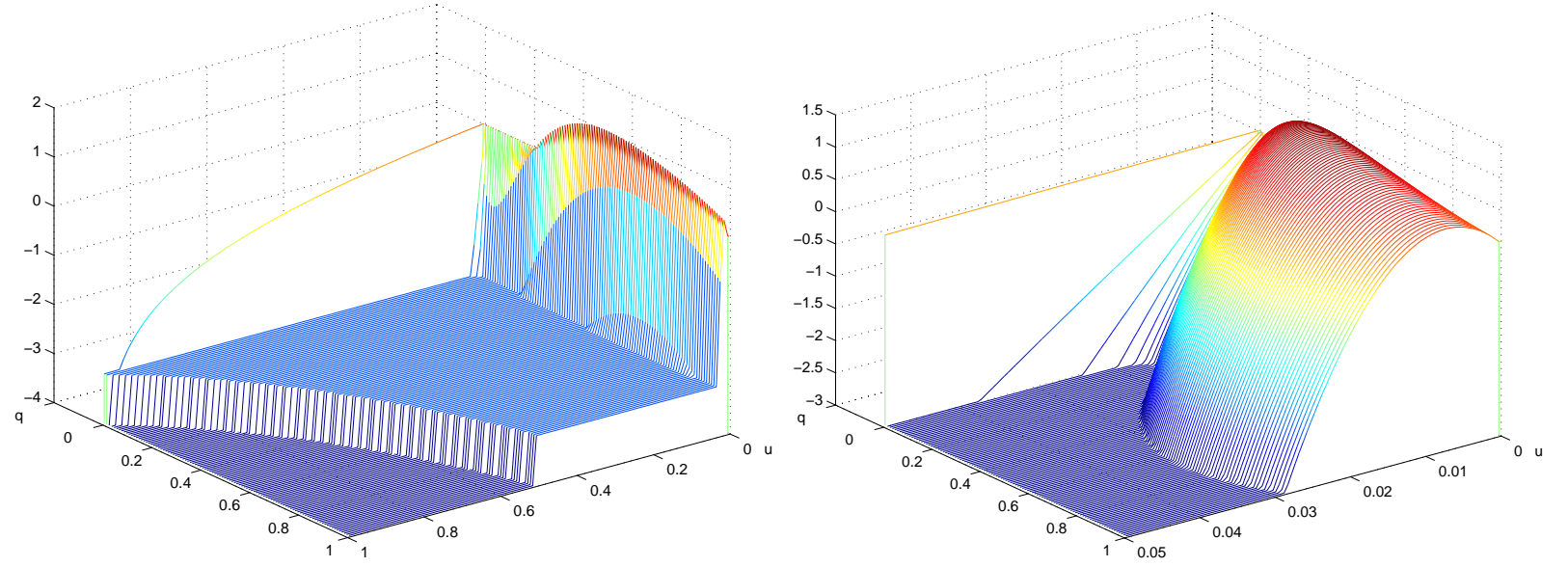

Figure 2. Case B. On left: on the whole domain $(q, u) \in[0,1] \times[0,1]$ by steps of 0.01 . On right: refinement $(q, u) \in[0,1] \times[0,0.05]$ by steps of $5.10^{-4}$ for $u$ and 0.01 for $q$.

\subsection{Distribution of $V_{n}(\eta)$}

The aim of this section is to study the rate in the distributional convergence of the LRT statistic on $\mathcal{P}_{\eta}$ to the asymptotic distribution given in Theorem 2.1:

$$
\frac{1}{2} \sup _{q \in \mathcal{Q}_{\eta}} \xi_{q}^{2} \cdot 1_{\xi_{q} \geq 0}
$$

to be able to say for how many observations the asymptotic approximation holds. We encounter difficulties to have a significant result on the whole $\mathcal{P}_{\eta}$ domain, so we do the simulations on a simplified model, where the supremum is taken over two values of $q$.

$$
\frac{1}{2} \sup _{q \in\{1,0.4\}} \xi_{q}^{2} \cdot 1_{\xi_{q} \geq 0}
$$

In this case, we can easily simulate the theoretical law, using the following property: if $X_{1}$ and $X_{2}$ are independent normal deviates $\mathcal{N}(0,1)$ and if

$$
Y_{1}=\sigma_{1} X_{1}, \quad Y_{2}=\sigma_{2}\left(\rho X_{1}+\sqrt{1-\rho^{2}} X_{2}\right)
$$

then $Y_{1}$ and $Y_{2}$ are dependent random variables, normally distributed with standard deviations $\sigma_{1}$ and $\sigma_{2}$, and with correlation coefficient $\rho$.

Figure 3 shows the histogram of the simulated law with 1000 points, with $\rho=0.18$ corresponding to the correlation when $q=1$ and $q=0.4$. The outline does not significantly change when we increase the number of points. Notice that the weight for $[0,0.05]$ is around 370 . For two independent normal deviates, this weight is around 340 , whereas for $\rho=1$, this weight is around 620 .

To approximate the distribution of the LRT statistic $V_{n}(0.4,1)$ in this case, we draw $m$ samples of length $n$, and compute for each sample the maximum likelihood ratio value for the two values of $q$. For this computation, we use a Newton algorithm, using the fact, checked on a great number of samples, that the log-likelihood has an unique maximum near $u=0$. Other simulations with an EM algorithm (Dempster et al. [12]) and various initial points did not give better results. We first take $m=500$ and $n=2000, n=5000, n=10000$ (Fig. 4 left). The weight of $[0,0.05]$ is around 250. Compared with the simulated law $370 / 2=185$, it is much more higher. If we now increase the number of samples to $m=5,000$ of size $n=10,000$ (Fig. 4 right), this weight $2450 / 10=245$ is not significantly modified. 


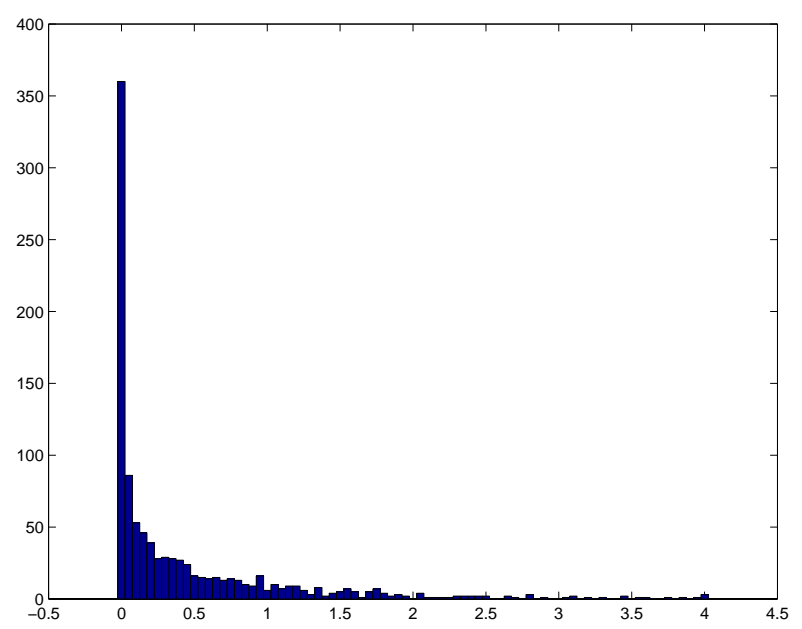

FigURE 3. Histogram of the simulated law $\frac{1}{2} \sup _{q \in\{1,0.4\}} \xi_{q}^{2} \cdot 1_{\xi_{q} \geq 0}$. with 1000 points.
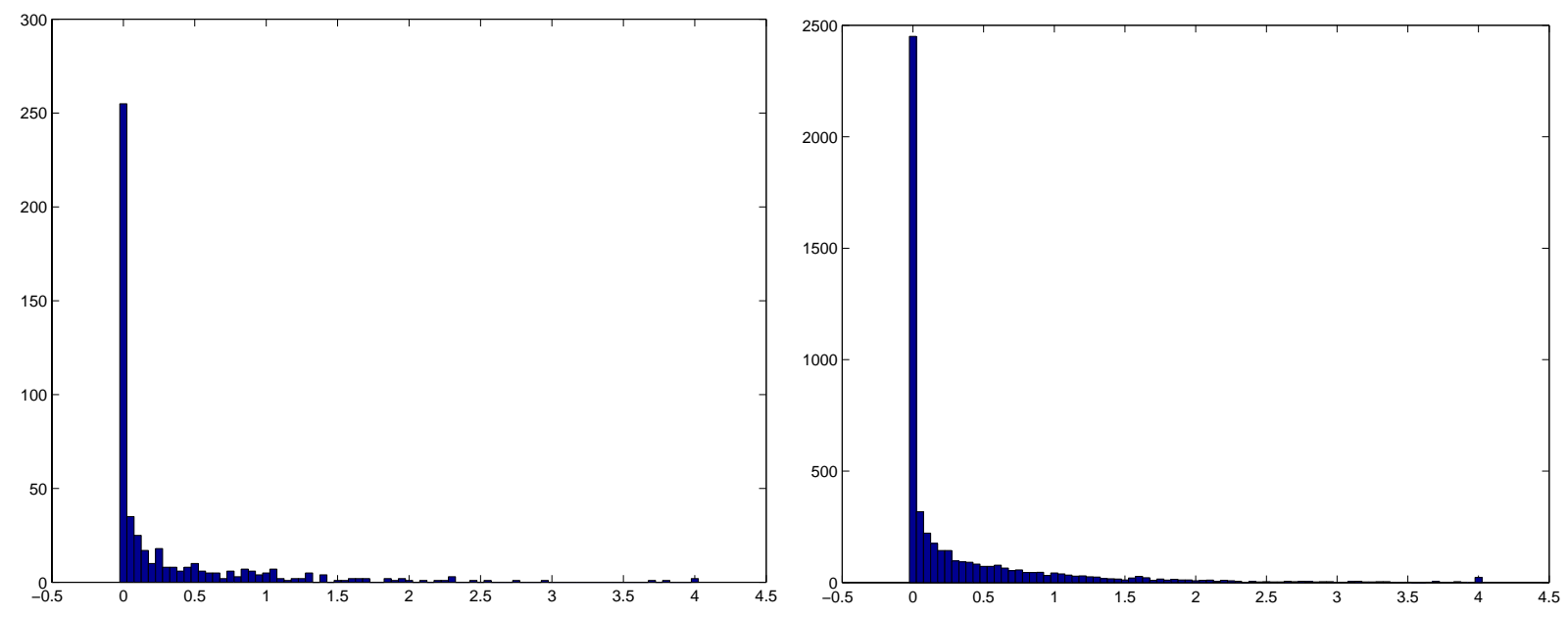

FIGURE 4. Histogram of the simulated LRT statistic $V_{n}(0.4,1), m=500$ (on left) and $m=5000$ (on right) samples of size $n=10000$.

If we increase the size of the samples to $n=100000$ for 500 samples (Fig. 5 left), this weight begins to decrease (around 230), and this is more significant for $m=50$ samples of size 1000000 (Fig. 5 right), where the weight is of the order of $20 \times 10=200$.

This allows us to conclude that the convergence is extremely slow, and we can relate this fact to the slow convergence of the LRT statistic for a mixture of two normal distributions as pointed out by Atwood et al. [1] or Chuang and Mendell [5]. Bootstrap techniques might also be another way to investigate the distribution, see McLachlan [28] or Feng and McCulloch [15] for the case of i.i.d mixtures.

\subsection{Convergence of the LRT statistic to infinity}

At which rate does the LRT on $\mathcal{P}_{0}$ converge to infinity? We numerically investigate the evolution of the LRT with the length $n$ of the sample. 

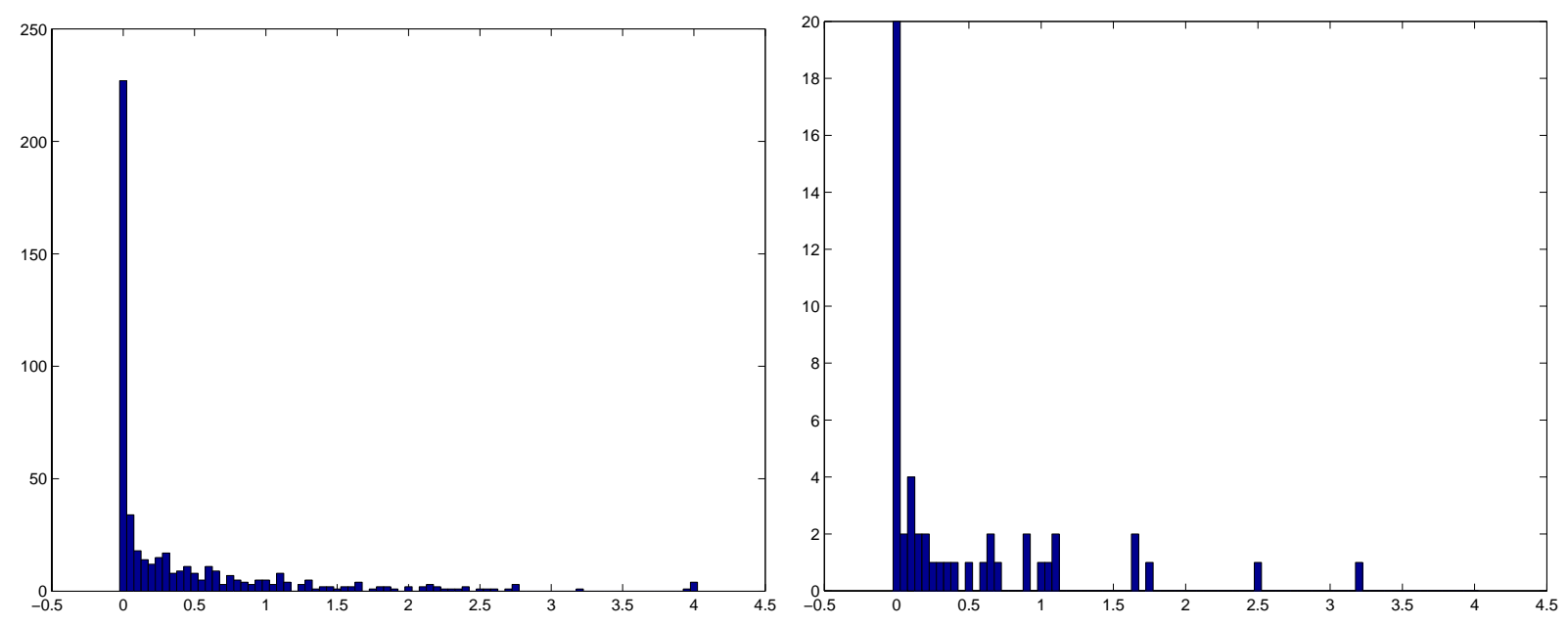

FIGURE 5. Histogram of the simulated LRT statistic $V_{n}(0.4,1), m=500$ samples of size $n=100000$ (on left) and $m=50$ samples of size $n=1000000$ (on right).

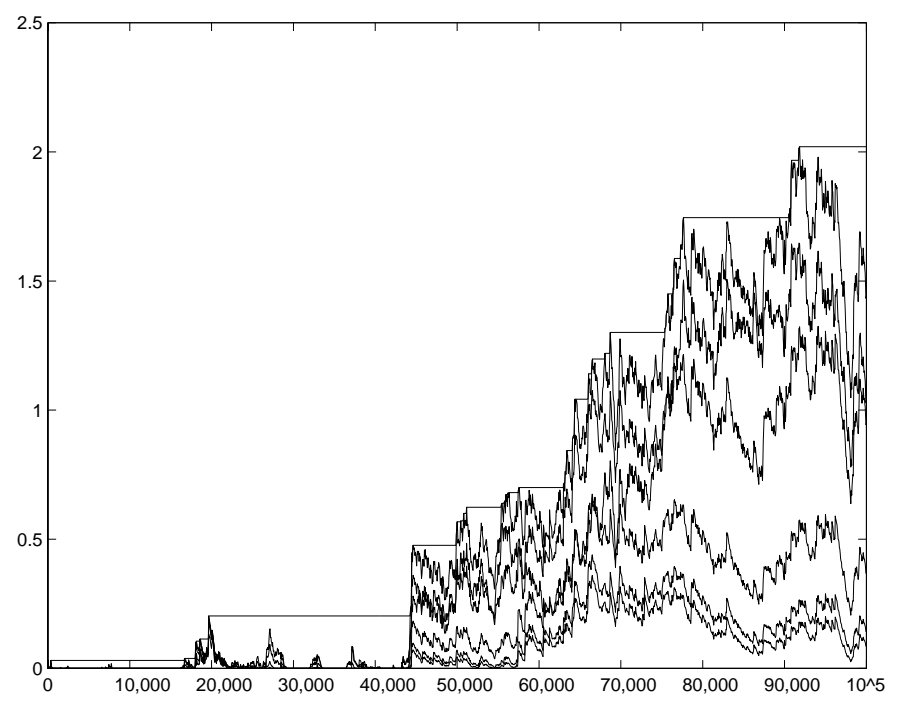

Figure $6 . V_{n}^{(1)}\left(q_{k}\right)$ for the six values of $q_{k}$ defined by $(17)$ with $\rho=0.85, k=0, \ldots, 5$. The sample size increases from size $n=50$ to $n=100000$ by steps of 50 .

As pointed out in the proof of Theorem 2.5, it is sufficient to take a sequence $q_{k}$ such that

$$
\left(1-q_{k}\right)^{2}\left(N^{2}+1\right)=1-\rho^{k^{2}}
$$

and observe that

tends to infinity with $n$, where

$$
\sup \left\{V_{n}^{(1)}\left(q_{k}\right): k \in \mathbb{N}\right\}
$$

$$
V_{n}^{(1)}\left(q_{k}\right)=\ell_{n}\left(\hat{u}_{q_{k}}, q_{k}\right)-\ell_{n}\left(0, q_{k}\right) .
$$

We choose $\rho=0.85, k=0, \ldots, 5$, and compute $V_{n}^{(1)}\left(q_{k}\right)$ from $n=50$ to $n=100000$ by steps of 50 (Fig. 6 ). The result shows that the convergence is very slow, but can be seen. For the i.i.d. mixture of two normals, 
Hartigan [19] gave a $\frac{1}{2} \log \log (n)$ rate. Such rate cannot be experimentally derived, it has to be theoretically investigated for mixtures with Markov regime in a further work.

\section{Proofs}

\subsection{Proof of Proposition $\mathbf{2 . 2}$}

Proposition 2.2 is a consequence of the following lemmas:

Lemma 4.1. Assume (A2). Let $(u, q)$ be a fixed parameter. Then under $H_{0},\left(Y_{k}, p_{k}(u, q)\right)_{k \in \mathbb{N}}$ is a uniformly ergodic chain with invariant probability $\pi=f_{\gamma_{0}} \nu \otimes \mu_{u, q}$. For $u=0, \mu_{0, q}$ is the dirac at 0 , and for $u \neq 0$,

$$
\int p d \mu_{u, q} \geq \frac{q u}{2(1-u)}
$$

Proof of Lemma 4.1. Under $H_{0},\left(Y_{k}, p_{k}(u, q)\right)_{k \in \mathbb{N}}$ is a Markov chain since the $\left(Y_{k}\right)$ are i.i.d. and by equation (8). Moreover, for any $k, Y_{k}$ and $p_{k}(u, q)$ are independent variables so that the lemma follows as soon as $\left(p_{k}(u, q)\right)_{k \in \mathbb{N}}$ is a uniformly ergodic chain with invariant probability $\mu_{u, q}$. For $u=0, p_{k}(0, q)=0$ for all $k$. For $u \neq 0,\left(p_{k}(u, q)\right)_{k \in \mathbb{N}}$ is a Feller chain, $\psi$-irreductible with $\psi$ the Lebesgue measure on $\left(\frac{q u}{1-u}, 1-q\right)$ thanks to (A2), thus a T-chain by Theorem 6.2.9, and then a uniformly ergodic chain by Theorem 16.2.5 of Meyn and Tweedie [31]. Now using equation (8) one obtains, using the fact that $p_{k}(u, q)$ is $\sigma\left(Y_{1}, \ldots, Y_{k-1}\right)$-measurable for all $k$, independent of $Y_{k}$, and using Jensen's inequality applied to the function $1 / x$ :

$$
E\left(p_{k+1}(u, q) / Y_{1}, \ldots, Y_{k-1}\right) \geq \frac{q u}{(1-u)}\left(1-p_{k}(u, q)\right)
$$

so that

$$
E\left(p_{k+1}(u, q)\right) \geq \frac{q u}{(1-u)}\left(1-E\left(p_{k}(u, q)\right)\right)
$$

and equation (18) follows by taking the limit as $k$ goes to infinity.

Lemma 4.2. Assume (A1) and (A2). Then under $H_{0}, S_{n}(u, q)$ converges a.s. to $S(u, q)$. Moreover, for any positive $\delta$,

$$
\sup _{u \geq \delta,(u, q) \in \mathcal{P}_{\eta}} S(u, q)=-2 \epsilon(\delta, \eta)<0 .
$$

Proof of Lemma 4.2. The a.s. convergence of $S_{n}(u, q)$ to $S(u, q)$ follows from the first point of Lemma 4.1. Now,

and by Jensen's inequality

$$
S(u, q)=\int f_{\gamma_{0}}(x) d \nu(x)\left[\int \log \left(1-p+p \frac{f_{\gamma_{1}}}{f_{\gamma_{0}}}(x)\right) d \mu_{u, q}(p)\right]
$$

$$
\int \log \left(1-p+p \frac{f_{\gamma_{1}}}{f_{\gamma_{0}}}(x)\right) d \mu_{u, q}(p) \leq \log \left(1-\int p d \mu_{u, q}(p)+\int p d \mu_{u, q}(p) \frac{f_{\gamma_{1}}}{f_{\gamma_{0}}}(x)\right)
$$

so that

$$
S(u, q) \leq-K\left(f_{\gamma_{0}},\left(1-\int p d \mu_{u, q}(p)\right) f_{\gamma_{0}}+\int p d \mu_{u, q}(p) f_{\gamma_{1}}\right) .
$$

Using now the second point of Lemma 4.1 one obtains

$$
\sup _{u \geq \delta,(u, q) \in \mathcal{P}_{\eta}} S(u, q) \leq-\inf \left\{K\left(f_{\gamma_{0}},(1-p) f_{\gamma_{0}}+p f_{\gamma_{1}}\right): u \geq \delta,(u, q) \in \mathcal{P}_{\eta}, p \geq \frac{q u}{2(1-u)}\right\} .
$$

Now using (A1) $K\left(f_{\gamma_{0}},(1-p) f_{\gamma_{0}}+p f_{\gamma_{1}}\right)$ is a continuous function of $p$ and the result follows. 
Lemma 4.3. There exist a positive number $M_{1}$ such that, for any integer $k$,

$$
E\left(\sup _{(u, q) \in \mathcal{P}_{\eta}}\left|\frac{\partial p_{k}}{\partial u}(u, q)\right|\right)<M_{1}, \quad E\left(\sup _{(u, q) \in \mathcal{P}_{\eta}}\left|\frac{\partial p_{k}}{\partial q}(u, q)\right|\right)<M_{1}
$$

Proof of Lemma 4.3. Let us give the iterative model for the first derivative with respect to $u$ and with respect to $q$ of $p_{k}(u, q)$ for any value of $(u, q)$. From now on, we shall denote by $W_{k}$ the random variable $\frac{f_{\gamma_{1}}\left(Y_{k}\right)}{f_{\gamma_{0}}\left(Y_{k}\right)}$. $W_{k}$ is non negative and its expectation under $H_{0}$ equals 1 . The initial value is

$$
\frac{\partial p_{1}}{\partial u}(u, q)=1, \frac{\partial p_{1}}{\partial q}(u, q)=0
$$

and for any positive integer $k$ :

$$
\begin{aligned}
& \frac{\partial p_{k+1}}{\partial u}(u, q)=\frac{q}{(1-u)^{2}} \frac{\left(1-p_{k}\right)}{\left(1-p_{k}\right)+p_{k} W_{k}}+\left(1-\frac{q}{(1-u)}\right) \frac{W_{k}}{\left[\left(1-p_{k}\right)+p_{k} W_{k}\right]^{2}} \frac{\partial p_{k}}{\partial u} \\
& \frac{\partial p_{k+1}}{\partial q}(u, q)=\frac{\frac{u}{(1-u)}\left(1-p_{k}\right)-p_{k} W_{k}}{\left(1-p_{k}\right)+p_{k} W_{k}}+\left(1-\frac{q}{(1-u)}\right) \frac{W_{k}}{\left[\left(1-p_{k}\right)+p_{k} W_{k}\right]^{2}} \frac{\partial p_{k}}{\partial q} .
\end{aligned}
$$

Direct computation leads to

$$
\left|\frac{\partial p_{k+1} / \partial u}{\left(1-p_{k+1}\right)^{2}}\right| \leq \frac{q}{(1-u)^{2}\left[\left(1-\frac{q u}{1-u}\right) \bigwedge q\right]^{2}}+\frac{1-\frac{q}{(1-u)}}{\left(1-\frac{q u}{(1-u)}\right)^{2}} W_{k}\left|\frac{\partial p_{k} / \partial u}{\left(1-p_{k}\right)^{2}}\right|
$$

in which $a \bigwedge b$ means $\inf \{a, b\}$. We have

$$
\sup \left\{\frac{q}{(1-u)^{2}\left[\left(1-\frac{q u}{1-u}\right) \bigwedge q\right]^{2}}:(u, q) \in \mathcal{P}_{\eta}\right\}=M_{2}<+\infty
$$

and also

so that

$$
\sup \left\{\frac{1-\frac{q}{(1-u)}}{\left(1-\frac{q u}{(1-u)}\right)^{2}}:(u, q) \in \mathcal{P}_{\eta}\right\}=1-h_{1}<1
$$

$$
\sup _{(u, q) \in \mathcal{P}_{\eta}}\left|\frac{\partial p_{k+1} / \partial u}{\left(1-p_{k+1}\right)^{2}}\right| \leq M_{2}+\left(1-h_{1}\right) W_{k} \sup _{(u, q) \in \mathcal{P}_{\eta}}\left|\frac{\partial p_{k} / \partial u}{\left(1-p_{k}\right)^{2}}\right|
$$

This leads to

$$
E\left[\sup _{(u, q) \in \mathcal{P}_{\eta}}\left|\frac{\partial p_{k+1} / \partial u}{\left(1-p_{k+1}\right)^{2}}\right|\right] \leq M_{2}+\left(1-h_{1}\right) E\left[\sup _{(u, q) \in \mathcal{P}_{\eta}}\left|\frac{\partial p_{k} / \partial u}{\left(1-p_{k}\right)^{2}}\right|\right]
$$

By induction one obtains that for any integer $k$

$$
E\left[\sup _{(u, q) \in \mathcal{P}_{\eta}}\left|\frac{\partial p_{k} / \partial u}{\left(1-p_{k}\right)^{2}}\right|\right] \leq \frac{M_{2}}{h_{1}}
$$

Since obviously

$$
\sup _{(u, q) \in \mathcal{P}_{\eta}}\left|\frac{\partial p_{k}}{\partial u}\right| \leq \sup _{(u, q) \in \mathcal{P}_{\eta}}\left|\frac{\partial p_{k} / \partial u}{\left(1-p_{k}\right)^{2}}\right|
$$


one obtains the first inequality of Lemma 4.3 with $M_{1}=\frac{M_{2}}{h_{1}}$. The second inequality may be obtained similarly.

Let us come back to the proof of Proposition 2.2. For any positive $\delta$, we have

$$
P\left(\sup _{q \in \mathcal{Q}_{\eta}} \widehat{u}_{q} \geq \delta\right) \geq P(\widehat{u} \geq \delta)
$$

so that it is enough to prove the proposition for $\sup _{q \in \mathcal{Q}_{\eta}} \widehat{u}_{q}$. Now, let $\left(u_{i}, q_{i}\right)$ be the centers of $N(h)$ squares of edge of lentgh $h$ covering $\mathcal{P}_{\eta}^{\delta}=\left\{(u, q) \in \mathcal{P}_{\eta}: u \geq \delta\right\}$. We have

$$
\begin{aligned}
P\left(\sup _{q \in \mathcal{Q}_{\eta}} \widehat{u}_{q} \geq \delta\right) & \leq P\left(\sup _{(u, q) \in \mathcal{P}_{\eta}^{\delta}} S_{n}(u, q) \geq 0\right) \leq \sum_{i=1}^{N(h)} P\left(S_{n}\left(u_{i}, q_{i}\right) \geq-\epsilon(\delta, \eta)\right) \\
& +P\left(\sup _{\left|u-u^{\prime}\right| \leq h,\left|q-q^{\prime}\right| \leq h,(u, q) \in \mathcal{P}_{\eta}^{\delta}}\left|S_{n}(u, q)-S_{n}\left(u^{\prime}, q^{\prime}\right)\right| \geq \epsilon(\delta, \eta)\right) .
\end{aligned}
$$

Now, as soon as $\left|u-u^{\prime}\right| \leq h,\left|q-q^{\prime}\right| \leq h,(u, q) \in \mathcal{P}_{\eta}^{\delta}$,

$$
\left|S_{n}(u, q)-S_{n}\left(u^{\prime}, q^{\prime}\right)\right| \leq \frac{h}{n} \sum_{k=1}^{n}\left(\sup _{(u, q) \in \mathcal{P}_{\eta}}\left|\frac{\partial p_{k}}{\partial u}(u, q)\right|+\sup _{(u, q) \in \mathcal{P}_{\eta}}\left|\frac{\partial p_{k}}{\partial q}(u, q)\right|\right) m\left(Y_{k}\right),
$$

where $m$ is the function

$$
m(x)=\max \left(\frac{f_{\gamma_{0}}}{f_{\gamma_{1}}}(x) ; \frac{f_{\gamma_{1}}}{f_{\gamma_{0}}}(x)\right)-1
$$

Use Lemma 4.1, Lemma 4.2, Markov inequality, the fact that $Y_{k}$ is independent of the $p_{k}$ and its derivatives, and Lemma 4.3, to conclude that

$$
\limsup _{n \rightarrow+\infty} P\left(\sup _{q \in \mathcal{Q}_{\eta}} \widehat{u}_{q} \geq \delta\right)=0
$$

and Proposition 2.2 follows.

\subsection{Proof of Proposition 2.3}

We shall first prove:

Lemma 4.4. Assume (A2, A3) and (A4). The random variable

$$
\frac{\partial^{2}}{\partial u^{2}} S_{n}(0, q)
$$

converges in probability to $N^{2} C(q, q)$ uniformly over $\mathcal{Q}_{\eta}$.

Proof of Lemma 4.4. We have from (13)

$$
\frac{\partial^{2}}{\partial u^{2}} S_{n}(0, q)=-\frac{1}{n} \sum_{k=1}^{n}\left(\frac{\partial p_{k}}{\partial u}(0, q)\right)^{2}\left(W_{k}-1\right)^{2}+\frac{1}{n} \sum_{k=1}^{n} \frac{\partial^{2} p_{k}}{\partial u^{2}}(0, q)\left(W_{k}-1\right) .
$$

We shall use the following trick (T1) for proving weak laws of large numbers for Markov chains. Let $\left(\Phi_{k}\right)_{k \in \mathbb{N}}$ be a Markov chain in $\mathbb{R}^{d}$ for some dimension $d$. Assume that $g$ is a norm-like function, that is a non negative function such that $\lim _{|x| \rightarrow+\infty} g(x)=+\infty$. 
1. If there exists an initial value $x$ such that

$$
\liminf _{k \rightarrow+\infty} E\left[g\left(\Phi_{k}\right) / \Phi_{0}=x\right]<+\infty
$$

then by Proposition 12.1.3 of Meyn and Tweedie [31], the Markov chain admits an invariant probability $\mu$.

2. If moreover for any initial points $v_{1}$ and $v_{2}, \Phi_{k}^{v_{1}}-\Phi_{k}^{v_{2}}$ tends in distribution to 0 , then by using 1. IV.22 of Duflo [14], the invariant probability is unique, and $\Phi_{k}$ converges in distribution to $\mu$.

3. Then, by using Proposition 6.4.2 of Meyn and Tweedie [31], $\left(\Phi_{k}\right)_{k \in \mathbb{N}}$ is an e-chain.

4. If moreover there exists a reachable state, then for any real function $f$ such that $f \leq V$,

$$
\frac{1}{n} \sum_{k=1}^{n} f\left(\Phi_{k}\right)
$$

converges in probability to $\int f d \mu$ by a similar proof as for Theorem 18.5.1 of Meyn and Tweedie [31]. We shall apply $(\mathbf{T 1})$ to $\Phi_{k}=\left(W_{k}, \frac{\partial p_{k}}{\partial u}(0, q), \frac{\partial^{2} p_{k}}{\partial u^{2}}(0, q)\right)$. Let us recall the recursive equations

$$
\begin{aligned}
\frac{\partial p_{k+1}}{\partial u}(0, q)= & q+(1-q) W_{k} \frac{\partial p_{k}}{\partial u}(0, q) \\
\frac{\partial^{2} p_{k+1}}{\partial u^{2}}(0, q)= & 2 q-2 q W_{k} \frac{\partial p_{k}}{\partial u}(0, q)-2(1-q)\left(W_{k}-1\right) W_{k}\left(\frac{\partial p_{k}}{\partial u}(0, q)\right)^{2} \\
& +(1-q) W_{k} \frac{\partial^{2} p_{k}}{\partial u^{2}}(0, q)
\end{aligned}
$$

with initialization $\frac{\partial p_{1}}{\partial u}(0, q)=1$ and $\frac{\partial^{2} p_{1}}{\partial u^{2}}(0, q)=0$. Notice that $\frac{\partial p_{k}}{\partial u}(u, q)$ is non negative for all integer $k$. (22) leads to

for any integer $k$, and

$$
E\left(\frac{\partial p_{k+1}}{\partial u}(0, q)\right)=1
$$

$$
E\left(\frac{\partial p_{k+1}}{\partial u}(0, q)\right)^{2}=q^{2}+2 q(1-q)+(1-q)^{2}\left(N^{2}+1\right) E\left(\frac{\partial p_{k}}{\partial u}(0, q)\right)^{2}
$$

so that by induction, for any integer $k$,

Thus

$$
E\left(\frac{\partial p_{k}}{\partial u}(0, q)\right)^{2} \leq \frac{2}{\eta}
$$

and, by induction, for any integer $k$,

$$
E\left|\frac{\partial^{2} p_{k+1}}{\partial u^{2}}(0, q)\right| \leq \frac{8}{\eta N^{2}}+(1-q) E\left|\frac{\partial^{2} p_{k}}{\partial u^{2}}(0, q)\right|
$$

$$
E\left|\frac{\partial^{2} p_{k+1}}{\partial u^{2}}(0, q)\right| \leq \frac{8}{\eta N^{2} q} .
$$

Point 1 of $(\mathbf{T} 1)$ holds with $g(x, y, z)=x^{2}+y^{2}+|z|$. To prove Point 2 , notice first that the $W_{k}$ are i.i.d. so that it is enough to prove it for the second and third coordinates of $\Phi_{k}$. Let $v_{1}=\left(y_{1}, z_{1}\right)$ and $v_{2}=\left(y_{2}, z_{2}\right)$ be two different initial points for $\left(\frac{\partial p_{k}}{\partial u}(0, q), \frac{\partial^{2} p_{k}}{\partial u^{2}}(0, q)\right)$. Let $\Delta_{k}\left(y_{1}, y_{2}\right)$ be the difference of $\frac{\partial p_{k}}{\partial u}(0, q)$ with initial values $y_{1}$ and $y_{2}$. Let now $S_{k}\left(y_{1}, y_{2}\right)$ be the sum of $\frac{\partial p_{k}}{\partial u}(0, q)$ with initial values $y_{1}$ and $y_{2}$, and $\Delta_{k}^{2}\left(v_{1}, v_{2}\right)$ be the difference of $\frac{\partial^{2} p_{k}}{\partial u^{2}}(0, q)$ when the initial values are $v_{1}$ and $v_{2}$. Equations (22) and (23) give

$$
\Delta_{k+1}\left(y_{1}, y_{2}\right)=(1-q) W_{k} \Delta_{k}\left(y_{1}, y_{2}\right)
$$




$$
\begin{gathered}
S_{k+1}\left(y_{1}, y_{2}\right)=2 q+(1-q) W_{k} S_{k}\left(y_{1}, y_{2}\right) \\
\Delta_{k+1}\left(y_{1}, y_{2}\right) S_{k+1}\left(y_{1}, y_{2}\right)=2 q(1-q) W_{k} \Delta_{k}\left(y_{1}, y_{2}\right)+(1-q)^{2} W_{k}^{2} \Delta_{k}\left(y_{1}, y_{2}\right) S_{k}\left(y_{1}, y_{2}\right) \\
\Delta_{k+1}^{2}\left(v_{1}, v_{2}\right)=-2 q W_{k} \Delta_{k}\left(y_{1}, y_{2}\right)-2(1-q)\left(W_{k}-1\right) W_{k} \Delta_{k}\left(y_{1}, y_{2}\right) S_{k}\left(y_{1}, y_{2}\right)+(1-q) W_{k} \Delta_{k}^{2}\left(v_{1}, v_{2}\right)
\end{gathered}
$$

By iterating these equations, one obtains that, for any integer $k$,

$$
\begin{gathered}
E\left|\Delta_{k}\left(y_{1}, y_{2}\right)\right|=(1-q)^{k-1}\left|y_{1}-y_{2}\right|, \\
E\left|\Delta_{k}\left(y_{1}, y_{2}\right) S_{k}\left(y_{1}, y_{2}\right)\right| \leq \frac{2 q}{\eta_{*}}(1-q)^{k-1}\left|y_{1}-y_{2}\right|,
\end{gathered}
$$

as soon as $\eta_{*}$ is such that $\eta_{*} \leq \eta$ and $\left|x_{1}^{2}-y_{1}^{2}\right| \leq 2 q / \eta_{*}$, and then

$$
E\left|\Delta_{k}^{2}\left(v_{1}, v_{2}\right)\right| \leq 2(1-q)^{k-1}\left|y_{1}-y_{2}\right|+\frac{4}{\eta_{*}}(1-q)^{k} N^{2}\left|y_{1}-y_{2}\right|
$$

as soon as also $\left|z_{1}-z_{2}\right| \leq 2\left|y_{1}-y_{2}\right|+\frac{4}{\eta_{*}}(1-q) N^{2}\left|y_{1}-y_{2}\right|$, which is always possible by choosing $\eta_{*}$ small enough. Consequently, $\Delta_{k}\left(y_{1}, y_{2}\right)$ and $\Delta_{k}^{2}\left(v_{1}, v_{2}\right)$ tend in distribution to 0 , and point 2 of (T1) holds. Since by using assumption (A2) the point $(0, q, 2 q)$ is obviously reachable, point 4 . also holds, and for any $q$ in $\mathcal{Q}_{\eta}$, the random variable

$$
\frac{\partial^{2}}{\partial u^{2}} S_{n}(0, q)
$$

converges in probability to $N^{2} C(q, q)$.

Let us now prove that this convergence is uniform. We have, using (22), for any $q$ and $q^{\prime}$ :

$$
\frac{\partial p_{k+1}}{\partial u}(0, q)-\frac{\partial p_{k+1}}{\partial u}\left(0, q^{\prime}\right)=\left(q-q^{\prime}\right)\left[1-W_{k} \frac{\partial p_{k}}{\partial u}(0, q)\right]+\left(1-q^{\prime}\right) W_{k}\left(\frac{\partial p_{k}}{\partial u}(0, q)-\frac{\partial p_{k}}{\partial u}\left(0, q^{\prime}\right)\right) .
$$

Using Lemma 4.3 one obtains by induction

$$
E \sup _{\left|q-q^{\prime}\right| \leq \delta, q, q^{\prime} \in \mathcal{Q}_{\eta}}\left|\frac{\partial p_{k}}{\partial u}(0, q)-\frac{\partial p_{k}}{\partial u}\left(0, q^{\prime}\right)\right| \leq \delta \cdot \frac{1+M_{1}}{q_{\eta}}
$$

Also,

$$
\left(\frac{\partial p_{k+1}}{\partial u}(0, q)\right)^{2}=q^{2}+2 q(1-q) W_{k} \frac{\partial p_{k}}{\partial u}(0, q)+(1-q)^{2} W_{k}^{2}\left(\frac{\partial p_{k}}{\partial u}(0, q)\right)^{2}
$$

so that,

$$
E \sup _{q \in \mathcal{Q}_{\eta}}\left(\frac{\partial p_{k}}{\partial u}(0, q)\right)^{2} \leq \frac{M_{1}+2}{\eta}
$$


Further, for any $q$ and $q^{\prime}$,

$$
\begin{aligned}
\left(\frac{\partial p_{k+1}}{\partial u}(0, q)\right)^{2}-\left(\frac{\partial p_{k+1}}{\partial u}\left(0, q^{\prime}\right)\right)^{2}= & q^{2}-q^{\prime 2}+\left(2 q(1-q)-2 q^{\prime}\left(1-q^{\prime}\right)\right) W_{k} \frac{\partial p_{k}}{\partial u}(0, q) \\
& 2 q^{\prime}\left(1-q^{\prime}\right) W_{k}\left(\frac{\partial p_{k}}{\partial u}(0, q)-\frac{\partial p_{k}}{\partial u}\left(0, q^{\prime}\right)\right) \\
& +\left((1-q)^{2}-\left(1-q^{\prime}\right)^{2}\right) W_{k}^{2}\left(\frac{\partial p_{k}}{\partial u}(0, q)\right)^{2} \\
& +\left(1-q^{\prime}\right)^{2} W_{k}^{2}\left[\left(\frac{\partial p_{k}}{\partial u}(0, q)\right)^{2}-\left(\frac{\partial p_{k}}{\partial u}\left(0, q^{\prime}\right)\right)^{2}\right]
\end{aligned}
$$

and by induction,

$$
E \sup _{\left|q-q^{\prime}\right| \leq \delta, q, q^{\prime} \in \mathcal{Q}_{\eta}}\left|\left(\frac{\partial p_{k}}{\partial u}(0, q)\right)^{2}-\left(\frac{\partial p_{k}}{\partial u}\left(0, q^{\prime}\right)\right)^{2}\right| \leq \delta \cdot M_{4}
$$

with

$$
M_{4}=\frac{1}{\eta} \cdot\left[2+2 M_{1}+\frac{1+M_{1}}{q_{\eta}}+\frac{2\left(N^{2}+1\right) M_{1}}{\eta}\right]
$$

Following the same lines, using (23) one obtains

$$
E \sup _{\left|q-q^{\prime}\right| \leq \delta, q, q^{\prime} \in \mathcal{Q}_{\eta}}\left|\frac{\partial^{2} p_{k}}{\partial u^{2}}(0, q)-\frac{\partial^{2} p_{k}}{\partial u^{2}}\left(0, q^{\prime}\right)\right| \leq \frac{\delta}{q_{\eta}} \cdot\left[2+2 M_{1}+2 \frac{1+M_{1}}{q_{\eta}}+2 N^{2}\left(\frac{M_{1}}{\eta}+M_{4}\right)\right] .
$$

Using (25) and (27), it follows that for some constant $M_{5}$ one has

$$
E \sup _{\left|q-q^{\prime}\right| \leq \delta, q, q^{\prime} \in \mathcal{Q}_{\eta}}\left|\frac{\partial^{2}}{\partial u^{2}} S_{n}(0, q)-\frac{\partial^{2}}{\partial u^{2}} S_{n}\left(0, q^{\prime}\right)\right| \leq \delta \cdot M_{5}
$$

and Lemma 4.4 follows.

To finish the proof of Proposition 2.3 we shall prove:

Lemma 4.5. Assume that (A2, A3) and (A4) hold. Then, for any random ũ converging to 0 uniformly in $q$,

$$
\frac{\partial^{2}}{\partial u^{2}} S_{n}(\tilde{u}, q)-\frac{\partial^{2}}{\partial u^{2}} S_{n}(0, q)
$$

converges in probability to 0 uniformly over $\mathcal{Q}_{\eta}$. 
Proof of Lemma 4.5. For any non negative $u$, we have

$$
\begin{aligned}
\frac{\partial^{2}}{\partial u^{2}} S_{n}(u, q)-\frac{\partial^{2}}{\partial u^{2}} S_{n}(0, q)= & \frac{1}{n} \sum_{k=1}^{n}\left(W_{k}-1\right)^{2}\left[\frac{\partial p_{k}}{\partial u}(0, q)^{2}-\frac{\partial p_{k}}{\partial u}(u, q)^{2}\right. \\
& \left.+\frac{\partial p_{k}}{\partial u}(u, q)^{2}\left(1-\frac{1}{\left(1-p_{k}(u, q)+p_{k}(u, q) W_{k}\right)^{2}}\right)\right] \\
& +\frac{1}{n} \sum_{k=1}^{n}\left(W_{k}-1\right)\left[-\frac{\partial^{2} p_{k}}{\partial u^{2}}(0, q)+\frac{\partial^{2} p_{k}}{\partial u^{2}}(u, q)\right. \\
& \left.+\frac{\partial^{2} p_{k}}{\partial u^{2}}(u, q)\left(\frac{1}{\left(1-p_{k}(u, q)+p_{k}(u, q) W_{k}\right)}-1\right)\right] .
\end{aligned}
$$

We shall use the following fact that holds as soon as $\bar{u}$ is small enough. For any integer $k$, any $q$ and any $u \leq \bar{u}$ :

$$
\frac{q u}{1-u} \leq p_{k}(u, q) \leq 1-q
$$

Also,

and

$$
1-\frac{1}{1-p_{k}(u, q)+p_{k}(u, q) W_{k}}=\frac{p_{k}(u, q)\left(W_{k}-1\right)}{1-p_{k}(u, q)+p_{k}(u, q) W_{k}}
$$

so that

$$
\left|\frac{1}{1-p_{k}(u, q)+p_{k}(u, q) W_{k}}\right| \leq \frac{1}{q_{\eta}}
$$

$$
\begin{aligned}
E\left(\sup _{q, u \leq \bar{u}}\left|\frac{\partial^{2}}{\partial u^{2}} S_{n}(u, q)-\frac{\partial^{2}}{\partial u^{2}} S_{n}(0, q)\right|\right) \leq & \frac{1}{n} \sum_{k=1}^{n}\left[N^{2} h_{1}(\bar{u})+\frac{1+q_{\eta}}{q_{\eta}^{2}} E\left|W_{k}-1\right|^{3} h_{2}(\bar{u})\right. \\
& \left.+E\left|W_{k}-1\right| h_{3}(\bar{u})+\frac{1}{q_{\eta}} E\left|W_{k}-1\right|^{2} h_{4}(\bar{u})\right]
\end{aligned}
$$

with

and

$$
\begin{gathered}
h_{1}(\bar{u}) \geq E\left(\sup _{q, u \leq \bar{u}}\left|\frac{\partial p_{k}}{\partial u}(0, q)^{2}-\frac{\partial p_{k}}{\partial u}(u, q)^{2}\right|\right), \\
h_{2}(\bar{u}) \geq E\left(\sup _{q, u \leq \bar{u}}\left|p_{k}(u, q) \frac{\partial p_{k}}{\partial u}(u, q)^{2}\right|\right), \\
h_{3}(\bar{u}) \geq E\left(\sup _{q, u \leq \bar{u}}\left|\frac{\partial^{2} p_{k}}{\partial u^{2}}(0, q)-\frac{\partial^{2} p_{k}}{\partial u^{2}}(u, q)\right|\right)
\end{gathered}
$$

$$
h_{4}(\bar{u}) \geq E\left(\sup _{q, u \leq \bar{u}}\left|p_{k}(u, q) \frac{\partial^{2} p_{k}}{\partial u^{2}}(u, q)\right|\right) .
$$

Lemma 4.5 will thus follow from the fact that it is possible to choose $h_{1}, h_{2}, h_{3}$ and $h_{4}$ such that for any $i=1, \ldots, 4, \lim _{u \rightarrow 0} h_{i}(u)=0$.

From now on, Cte will designate a universal constant number. We shall use the fact (T2) that if the deterministic real valued sequence $\left(z_{k}\right)_{k \in \mathbb{N}}$ satisfies, for a positive number $\delta$, for any integer $k$

$$
z_{k+1} \leq M+(1-\delta) z_{k}
$$

and also $z_{1} \leq M$, then for any integer $k$

$$
z_{k} \leq \mathrm{Cte} \cdot M
$$


(T2) will often be used with $M$ involving $\bar{u}$, and tending to 0 when $\bar{u}$ does.

Lemma 4.6. As soon as $\bar{u}$ is small enough,

$$
E\left(\sup _{q, u \leq \bar{u}} \frac{\partial p_{k}}{\partial u}(u, q)^{2}\right) \leq \text { Cte }<+\infty
$$

Proof of Lemma 4.6. Direct computation leads to, for any integer $k$, any $q$ and any $u \leq \bar{u}$ :

$$
\begin{aligned}
\frac{1}{\left(1-p_{k+1}(u, q)\right)^{4}}\left(\frac{\partial p_{k+1}}{\partial u}(u, q)\right)^{2} \leq & \frac{1}{q^{2}(1-\bar{u})^{4}}+\frac{2}{q^{4}(1-\bar{u})^{2}}(1-q) W_{k} \frac{\partial p_{k}}{\partial u}(u, q) \\
& \frac{\left(1-\frac{q}{1-u}\right)^{2}}{\left(1-\frac{q u}{1-u}\right)^{4}} W_{k}^{2} \frac{1}{\left(1-p_{k}(u, q)\right)^{4}}\left(\frac{\partial p_{k}}{\partial u}(u, q)\right)^{2} .
\end{aligned}
$$

Now there exists $\bar{u}$ small enough such that:

$$
\sup _{q, u \leq \bar{u}} \frac{\left(1-\frac{q}{1-u}\right)^{2}}{\left(1-\frac{q u}{1-u}\right)^{4}} E\left(W_{k}^{2}\right) \leq 1-\frac{\eta}{2}
$$

Taking the supremum over $q$ and $u \leq \bar{u}$, then taking expectation, and then using (T2) one obtains that for any integer $k$ :

$$
E\left[\sup _{q, u \leq \bar{u}} \frac{1}{\left(1-p_{k}(u, q)\right)^{4}}\left(\frac{\partial p_{k}}{\partial u}(u, q)\right)^{2}\right] \leq \text { Cte }<+\infty
$$

and Lemma 4.6 follows:

Lemma 4.7. As soon as $\bar{u}$ is small enough,

$$
E\left(\sup _{q, u \leq \bar{u}} \frac{\partial p_{k}}{\partial u}(u, q)^{2+\epsilon_{*}}\right) \leq \mathrm{Cte}<+\infty
$$

in which $\epsilon_{*}$ is defined below.

Proof of Lemma 4.7. Using (A4),

$$
3^{\epsilon} \sup _{q \in \mathcal{Q}_{\eta}}(1-q)^{2+\epsilon} E\left(W_{k}\right)^{2+\epsilon}
$$

is a finite, continuous function of $(q, \epsilon)$ for $\epsilon<\epsilon_{0}$, which is upper bounded by $1-\eta$ for $\epsilon=0$, so that there exists a positive $\epsilon_{*}$ such that

$$
3^{\epsilon_{*}} \sup _{q \in \mathcal{Q}_{\eta}}(1-q)^{2+\epsilon_{*}} E\left(W_{k}\right)^{2+\epsilon_{*}} \leq 1-\eta / 2 .
$$


We now have for any integer $k$, any $q$ and any $u \leq \bar{u}$ :

$$
\begin{aligned}
\left(\frac{1}{\left(1-p_{k+1}(u, q)\right)^{2}} \frac{\partial p_{k+1}}{\partial u}(u, q)\right)^{2+\epsilon_{*}} & =\left(\frac{1}{\left(1-p_{k+1}(u, q)\right)^{4}} \frac{\partial p_{k+1}}{\partial u}(u, q)^{2}\right)^{1+\epsilon_{*} / 2} \\
\leq & \left(\operatorname{Cte}+\operatorname{Cte} W_{k} \frac{\partial p_{k}}{\partial u}(u, q)\right. \\
& \left.+\frac{\left(1-\frac{q}{1-u}\right)^{2}}{\left(1-\frac{q u}{1-u}\right)^{4}} W_{k}^{2} \frac{1}{\left(1-p_{k}(u, q)\right)^{4}} \frac{\partial p_{k}}{\partial u}(u, q)^{2}\right)^{1+\epsilon_{*} / 2} \\
\leq & 3^{\epsilon_{*} / 2}\left(\operatorname{Cte}+\operatorname{Cte} W_{k}^{1+\epsilon_{*} / 2} \frac{\partial p_{k}}{\partial u}(u, q)^{1+\epsilon_{*} / 2}\right. \\
& \left.+\frac{\left(1-\frac{q}{1-u}\right)^{2+\epsilon_{*}}}{\left(1-\frac{q u}{1-u}\right)^{4+2 \epsilon_{*}}} W_{k}^{2+\epsilon_{*}}\left(\frac{1}{\left(1-p_{k}(u, q)\right)^{4}} \frac{\partial p_{k}}{\partial u}(u, q)^{2}\right)^{1+\epsilon_{*} / 2}\right) .
\end{aligned}
$$

Now there exists $\bar{u}$ small enough such that:

$$
\sup _{q, u \leq \bar{u}} 3^{\epsilon_{*}} \frac{\left(1-\frac{q}{1-u}\right)^{2+\epsilon_{*}}}{\left(1-\frac{q u}{1-u}\right)^{4+2 \epsilon_{*}}} E\left(W_{k}^{2+\epsilon_{*}}\right) \leq 1-\frac{\eta}{4}
$$

Taking the supremum over $q$ and $u \leq \bar{u}$, then taking expectation, and then using (T2) one obtains that for any integer $k$ :

and Lemma 4.7 follows:

$$
E\left[\sup _{q, u \leq \bar{u}} \frac{1}{\left(1-p_{k}(u, q)\right)^{4+2 \epsilon_{*}}}\left(\frac{\partial p_{k}}{\partial u}(u, q)\right)^{2+\epsilon_{*}}\right] \leq \mathrm{Cte}<+\infty
$$

Lemma 4.8. As soon as $\bar{u}$ is small enough,

$$
E\left(\sup _{q, u \leq \bar{u}} p_{k}(u, q)\right) \leq \mathrm{Cte} \cdot \bar{u} .
$$

Proof of Lemma 4.8. Direct computation leads to, for any integer $k$, any $q$ and any $u \leq \bar{u}$ :

$$
\frac{p_{k+1}(u, q)}{1-p_{k+1}(u, q)} \leq \mathrm{Cte} \cdot \bar{u}+\frac{1-q_{\eta}}{1-\frac{u}{1-u}} W_{k} \frac{p_{k}(u, q)}{1-p_{k}(u, q)} .
$$

Notice that $p_{1}(u, q)=u$. Taking the supremum over $q$ and $u \leq \bar{u}$, then taking expectation, and then using (T2) one obtains Lemma 4.8 .

Lemma 4.9. As soon as $\bar{u}$ is small enough, it is possible to choose $h_{2}$ such that

$$
\lim _{u \rightarrow 0} h_{2}(u)=0 .
$$

Proof of Lemma 4.9. Using Minkowsky's inequality, one may choose

$$
h_{2}(\bar{u}) \leq E\left(\left(\sup _{q, u \leq \bar{u}} p_{k}(u, q)\right)^{1+2 / \epsilon_{*}}\right)^{1 /\left(1+2 / \epsilon_{*}\right)} E\left(\sup _{q, u \leq \bar{u}} \frac{\partial p_{k}}{\partial u}(u, q)^{2+\epsilon_{*}}\right)^{1 /\left(1+\epsilon_{*} / 2\right)} .
$$

Since the $p_{k}$ are $\leq 1$, we have for any integer $k$, any $q$ and any $u$

$$
\left.a p_{k}(u, q)\right)^{1+2 / \epsilon_{*}} \leq p_{k}(u, q)
$$


and using Lemma 4.7 and Lemma 4.8 one may choose

$$
h_{2}(\bar{u})=\operatorname{Cte} \cdot(\bar{u})^{1 /\left(1+2 / \epsilon_{*}\right)} .
$$

Lemma 4.10. As soon as $\bar{u}$ is small enough, it is possible to choose $h_{1}$ such that

$$
\lim _{u \rightarrow 0} h_{1}(u)=0 \text {. }
$$

Proof of Lemma 4.10. Direct computation leads to, for any integer $k$, any $q$ and any $u \leq \bar{u}$ :

$$
\begin{aligned}
\left|\frac{\partial p_{k+1}}{\partial u}(u, q)-\frac{\partial p_{k+1}}{\partial u}(0, q)\right| & \leq \mathrm{Cte} \cdot \bar{u}+\mathrm{Cte} \cdot W_{k} p_{k}(u, q)+\mathrm{Cte} \cdot \bar{u} W_{k} \frac{\partial p_{k}}{\partial u}(u, q) \\
& +\mathrm{Cte} \cdot W_{k}^{2} p_{k}(u, q) \frac{\partial p_{k}}{\partial u}(u, q)+(1-q) W_{k}\left|\frac{\partial p_{k}}{\partial u}(u, q)-\frac{\partial p_{k}}{\partial u}(0, q)\right| .
\end{aligned}
$$

Taking the supremum over $q$ and $u \leq \bar{u}$, then taking expectation, and then using (T2), Lemma 4.3 and Lemma 4.8, one obtains that for any integer $k$,

$$
E\left(\sup _{q, u \leq \bar{u}}\left|\frac{\partial p_{k}}{\partial u}(u, q)-\frac{\partial p_{k}}{\partial u}(0, q)\right|\right) \leq h_{5}(\bar{u})
$$

with

$$
h_{5}(u)=\frac{1}{q_{\eta}} \cdot \mathrm{Cte} \cdot u
$$

Now, since

$$
\left|\frac{\partial p_{k}}{\partial u}(u, q)^{2}-\frac{\partial p_{k}}{\partial u}(0, q)^{2}\right|=\left|\frac{\partial p_{k}}{\partial u}(u, q)-\frac{\partial p_{k}}{\partial u}(0, q)\right| \cdot\left(\frac{\partial p_{k}}{\partial u}(u, q)+\frac{\partial p_{k}}{\partial u}(0, q)\right)
$$

direct computation leads to, for any integer $k$, any $q$ and any $u \leq \bar{u}$ :

$$
\begin{aligned}
\left|\frac{\partial p_{k+1}}{\partial u}(u, q)^{2}-\frac{\partial p_{k+1}}{\partial u}(0, q)^{2}\right| & \leq \mathrm{Cte} \cdot \bar{u}+\mathrm{Cte} \cdot \bar{u} \sup _{q, u \leq \bar{u}} \frac{\partial p_{k}}{\partial u}(u, q) \\
& +\mathrm{Cte} \cdot W_{k} \sup _{q, u \leq \bar{u}} p_{k}(u, q) \sup _{q, u \leq \bar{u}} \frac{\partial p_{k}}{\partial u}(u, q) \\
& +\mathrm{Cte} \cdot \bar{u} W_{k} \sup _{q, u \leq \bar{u}} \frac{\partial p_{k}}{\partial u}(u, q)^{2} \\
& +\mathrm{Cte} \cdot W_{k}^{2} \sup _{q, u \leq \bar{u}} p_{k}(u, q) \frac{\partial p_{k}}{\partial u}(u, q)^{2} \\
& +\mathrm{Cte} \cdot W_{k}\left|\frac{\partial p_{k}}{\partial u}(u, q)-\frac{\partial p_{k}}{\partial u}(0, q)\right| \\
& +\mathrm{Cte} \cdot \bar{u} W_{k}^{2} \frac{\partial p_{k}}{\partial u}(u, q)^{2} \\
& +\mathrm{Cte} \cdot W_{k}^{3} \sup _{q, u \leq \bar{u}} p_{k}(u, q) \sup _{q, u \leq \bar{u}} \frac{\partial p_{k}}{\partial u}(u, q)^{2} \\
& +(1-q)^{2} W_{k}^{2}\left|\frac{\partial p_{k}}{\partial u}(u, q)^{2}-\frac{\partial p_{k}}{\partial u}(0, q)^{2}\right|
\end{aligned}
$$

Taking the supremum over $q$ and $u \leq \bar{u}$, then taking expectation, and then using (T2), Lemma 4.6, Lemma 4.8, equation (30) and equation (31) one obtains Lemma 4.10. 
Lemma 4.11. As soon as $\bar{u}$ is small enough,

$$
E\left(\sup _{q, u \leq \bar{u}}\left|\frac{\partial^{2} p_{k}}{\partial u^{2}}(u, q)\right|\right) \leq \text { Cte }<+\infty .
$$

Proof of Lemma 4.11. First of all, let us give the recursive equations for the second derivative of the prediction filter:

$$
\begin{aligned}
\frac{\partial^{2} p_{k+1}}{\partial u^{2}}(u, q)= & \frac{2 q}{(1-u)^{3}} \frac{\left(1-p_{k}\right)}{\left(1-p_{k}\right)+p_{k} W_{k}} \\
& -\frac{2 q}{(1-u)^{2}} \frac{W_{k}}{\left[\left(1-p_{k}\right)+p_{k} W_{k}\right]^{2}} \frac{\partial p_{k}}{\partial u}(u, q) \\
-2\left(1-\frac{q}{(1-u)}\right) \cdot & \left(W_{k}-1\right) \frac{W_{k}}{\left[\left(1-p_{k}\right)+p_{k} W_{k}\right]^{3}}\left(\frac{\partial p_{k}}{\partial u}(u, q)\right)^{2} \\
& +\left(1-\frac{q}{(1-u)}\right) \frac{W_{k}}{\left[\left(1-p_{k}\right)+p_{k} W_{k}\right]^{2}} \frac{\partial^{2} p_{k}}{\partial u^{2}}(u, q)
\end{aligned}
$$

with initial value $\frac{\partial^{2} p_{1}}{\partial u^{2}}(u, q)=0$. Direct computation leads to, for any integer $k$, any $q$ and any $u \leq \bar{u}$ :

$$
\begin{aligned}
\frac{1}{\left(1-p_{k+1}(u, q)\right)^{2}}\left|\frac{\partial^{2} p_{k+1}}{\partial u^{2}}(u, q)\right| & \leq \mathrm{Cte}+\mathrm{Cte} \cdot W_{k} \frac{\partial p_{k}}{\partial u}(u, q)+\mathrm{Cte} \cdot W_{k}^{2} \frac{\partial p_{k}}{\partial u}(u, q)^{2} \\
& +\frac{\left(1-\frac{q}{1-u}\right)}{\left(1-\frac{q u}{1-u}\right)^{2}} W_{k} \frac{1}{\left(1-p_{k}(u, q)\right)^{2}}\left|\frac{\partial^{2} p_{k}}{\partial u^{2}}(u, q)\right| .
\end{aligned}
$$

Now there exists $\bar{u}$ small enough such that:

$$
\sup _{q, u \leq \bar{u}} \frac{\left(1-\frac{q}{1-u}\right)}{\left(1-\frac{q u}{1-u}\right)^{2}} \leq 1-\frac{\eta}{2}
$$

Taking the supremum over $q$ and $u \leq \bar{u}$, then taking expectation, and then using (T2) one obtains that for any integer $k$ :

$$
E\left[\sup _{q, u \leq \bar{u}} \frac{1}{\left(1-p_{k}(u, q)\right)^{2}}\left|\frac{\partial^{2} p_{k}}{\partial u^{2}}(u, q)\right|\right] \leq \text { Cte }<+\infty
$$

and Lemma 4.11 follows:

Lemma 4.12. As soon as $\bar{u}$ is small enough, it is possible to choose $h_{4}$ such that

$$
\lim _{u \rightarrow 0} h_{4}(u)=0
$$


Proof of Lemma 4.12. Follows from the recursive inequality.

$$
\begin{aligned}
\frac{p_{k+1}(u, q)}{\left(1-p_{k+1}(u, q)\right)^{3}}\left|\frac{\partial^{2} p_{k+1}}{\partial u^{2}}(u, q)\right| & \leq \mathrm{Cte} \cdot p_{k+1}(u, q)+\mathrm{Cte} \cdot \bar{u} \cdot W_{k} \frac{\partial p_{k}}{\partial u}(u, q) \\
& +\mathrm{Cte} \cdot \bar{u} W_{k}^{2} \frac{\partial p_{k}}{\partial u}(u, q)^{2} \\
& +\mathrm{Cte} \cdot W_{k}^{2} p_{k}(u, q) \frac{\partial p_{k}}{\partial u}(u, q) \\
& +\mathrm{Cte} \cdot W_{k}^{3} p_{k}(u, q) \frac{\partial p_{k}}{\partial u}(u, q)^{2} \\
& +\mathrm{Cte} \cdot \bar{u} W_{k}\left|\frac{\partial^{2} p_{k}}{\partial u^{2}}(u, q)\right| \\
& +\frac{\left(1-\frac{q}{1-u}\right)^{2}}{\left(1-\frac{q u}{1-u}\right)^{3}} W_{k}^{2} \frac{p_{k}(u, q)}{\left(1-p_{k}(u, q)\right)^{3}}\left|\frac{\partial^{2} p_{k}}{\partial u^{2}}(u, q)\right| .
\end{aligned}
$$

Lemma 4.13. As soon as $\bar{u}$ is small enough, it is possible to choose $h_{3}$ such that

$$
\lim _{u \rightarrow 0} h_{3}(u)=0 \text {. }
$$

Proof of Lemma 4.13. Follows from the recursive inequality:

$$
\begin{aligned}
\left|\frac{\partial^{2} p_{k+1}}{\partial u^{2}}(u, q)-\frac{\partial^{2} p_{k+1}}{\partial u^{2}}(0, q)\right| & \leq \mathrm{Cte} \cdot \bar{u}+\mathrm{Cte} \cdot W_{k} p_{k}(u, q) \\
& +\mathrm{Cte} \cdot W_{k}\left|\frac{\partial p_{k}}{\partial u}(u, q)-\frac{\partial p_{k}}{\partial u}(0, q)\right| \\
& +\mathrm{Cte} \cdot \bar{u} W_{k} \frac{\partial p_{k}}{\partial u}(u, q)+\mathrm{Cte} \cdot W_{k}^{2} p_{k}(u, q) \frac{\partial p_{k}}{\partial u}(u, q) \\
& +\mathrm{Cte} \cdot W_{k}^{2}\left|\frac{\partial p_{k}}{\partial u}(u, q)^{2}-\frac{\partial p_{k}}{\partial u}(0, q)^{2}\right| \\
& +\mathrm{Cte} \cdot \bar{u} W_{k}^{2} \frac{\partial p_{k}}{\partial u}(u, q)^{2} \\
& +\mathrm{Cte} \cdot W_{k}^{3} p_{k}(u, q) \frac{\partial p_{k}}{\partial u}(u, q)^{2} \\
& +\mathrm{Cte} \cdot \bar{u} W_{k}\left|\frac{\partial^{2} p_{k}}{\partial u^{2}}(u, q)\right| \\
& +\mathrm{Cte} \cdot W_{k}^{2} p_{k}(u, q)\left|\frac{\partial^{2} p_{k}}{\partial u^{2}}(u, q)\right| \\
& +(1-q) W_{k}\left|\frac{\partial^{2} p_{k}}{\partial u^{2}}(u, q)-\frac{\partial^{2} p_{k}}{\partial u^{2}}(0, q)\right| .
\end{aligned}
$$

\subsection{Proof of Proposition 2.4}

To prove Proposition 2.4, we shall prove the convergence of finite marginal distributions of the process and its tightness. We shall use:

Lemma 4.14. Under the assumptions of Proposition 2.4, for any $q$ in $\mathcal{Q}_{\eta}$,

$$
\left(\frac{\partial p_{k}}{\partial u}(0, q) ; W_{k}\right)_{k \in \mathbb{N}}
$$


is a positive Harris Markov chain, g-uniformly ergodic for $g(x)=1+\|x\|_{2}^{2+\epsilon_{*}}$.

Proof of Lemma 4.14. It is enough to prove the results of the lemma for the Markov chain $\Phi=\left(\frac{\partial p_{k}}{\partial u}(0, q)\right)_{k \in \mathbb{N}}$. Using (A2), and applying Proposition 4.2.1 of Meyn and Tweedie [31], $\Phi$ is $\psi$-irreducible with $\psi$ the Lebesgue measure on $[q,+\infty[$. Then, since $\Phi$ is a Feller chain by (15), applying Propositions 6.2.8 and 6.2.9 of Meyn and Tweedie [31], all compact subsets of $[q,+\infty[$ are petite and $\Phi$ is a T-chain. Also, $\Phi$ is strongly aperiodic. Taking now $g(x)=1+\|x\|_{2}^{2+\epsilon_{*}}$, we have

$$
\begin{aligned}
E\left(V\left(\frac{\partial p_{k+1}}{\partial u}(0, q)\right) / \frac{\partial p_{k}}{\partial u}(0, q)=p\right)= & E\left(\left(q+(1-q) \frac{f_{\gamma_{1}}}{f_{\gamma_{0}}}(Y) p\right)^{2}\right)^{1+\epsilon_{*} / 2}+1 \\
\leq & 1+3^{\epsilon_{*}}\left(q^{2+\epsilon_{*}}\right. \\
& +[2 q(1-q)]^{1+\epsilon_{*} / 2} E\left(\frac{f_{\gamma_{1}}}{f_{\gamma_{0}}}(Y)\right)^{1+\epsilon_{*} / 2} p^{1+\epsilon_{*} / 2} \\
& \left.+(1-q)^{2+\epsilon_{*}} E\left(\frac{f_{\gamma_{1}}}{f_{\gamma_{0}}}(Y)\right)^{2+\epsilon_{*}}(g(p)-1)\right) .
\end{aligned}
$$

The right side of the last inequality divided by $V(p)$ tends to

$$
3^{\epsilon_{*}}(1-q)^{2+\epsilon_{*}} E\left(\frac{f_{\gamma_{1}}}{f_{\gamma_{0}}}(Y)\right)^{2+\epsilon_{*}}
$$

as $p$ tends to $\infty$, which is less or equal to $1-\eta / 2$, so that there exists a $M$ such that, for $p \geq M$,

$$
E\left(V\left(\frac{\partial p_{k+1}}{\partial u}(0, q)\right) / \frac{\partial p_{k}}{\partial u}(0, q)=p\right) \leq(1-\eta / 3) g(p),
$$

and we have for some positive $b$,

$$
E\left(g\left(\frac{\partial p_{k+1}}{\partial u}(0, q)\right) / \frac{\partial p_{k}}{\partial u}(0, q)=p\right)-g(p) \leq-\eta / 3 \cdot g(p)+b 1_{p \leq M}
$$

Consequently $\Phi$ is Harris positive by Theorem 11.3.4 and $V$-uniformly ergodic by Theorem 16.1.2 of Meyn and Tweedie [31].

Lemma 4.15. For any integer $m$ and any $q_{1}, \ldots, q_{m}$ in $\mathcal{B}_{\eta}$, the vector

$$
\sqrt{n}\left(\frac{\partial}{\partial u} S_{n}\left(0, q_{1}\right), \ldots, \frac{\partial}{\partial u} S_{n}\left(0, q_{m}\right)\right)
$$

converges in distribution to the centered Gaussian distribution with covariance $N^{2} C\left(q_{i}, q_{j}\right)$.

Proof of Lemma 4.15. Define

$$
M_{n}=n\left(\frac{\partial}{\partial u} S_{n}\left(0, q_{1}\right), \ldots, \frac{\partial}{\partial u} S_{n}\left(0, q_{m}\right)\right) .
$$

$M_{n}$ is a martingale with respect to the $\sigma$-field $\mathcal{F}_{n}=\sigma\left(Y_{1}, \ldots, Y_{n}\right)$, with bracket

$$
\left\langle M_{n}\right\rangle_{i, j}=N^{2} \sum_{k=1}^{n} \frac{\partial p_{k}}{\partial u}\left(0, q_{i}\right) \frac{\partial p_{k}}{\partial u}\left(0, q_{j}\right) .
$$


We shall apply Theorem 2.8.41 of Dacunha-Castelle and Duflo [8]. The Lindeberg condition holds since, for any positive $\delta$,

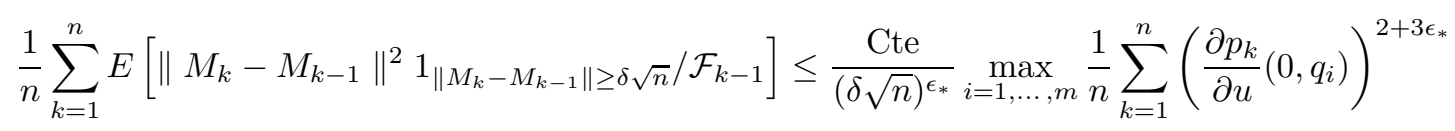

which converges to 0 as $n$ tends to infinity thanks to Lemma 4.14. Theorem 1.IV.24 of Duflo [14] may be applied (with $a=2$ and $\alpha=1-\eta)$ to the iterative markovian model leading to $\left(\frac{\partial p_{k}}{\partial u}\left(0, q_{1}\right), \ldots, \frac{\partial p_{k}}{\partial u}\left(0, q_{m}\right)\right.$ ), so that

$$
\frac{1}{n} \sum_{k=1}^{n} \frac{\partial p_{k}}{\partial u}\left(0, q_{i}\right) \frac{\partial p_{k}}{\partial u}\left(0, q_{j}\right)
$$

converges, as $n$ tends to infinity, to the covariance of $\frac{\partial p_{k}}{\partial u}\left(0, q_{i}\right)$ and $\frac{\partial p_{k}}{\partial u}\left(0, q_{j}\right)$ with respect to the invariant distribution of the Markov chain. This covariance with respect to the invariance distribution may be computed using equation (15). The obtained limiting value is

$$
\frac{1-\left(1-q_{1}\right)\left(1-q_{2}\right)}{1-\left(1-q_{1}\right)\left(1-q_{2}\right)\left(N^{2}+1\right)}
$$

so that

and Lemma 4.15 follows:

$$
\lim _{n \rightarrow+\infty} \frac{1}{n}\left\langle M_{n}\right\rangle_{i, j}=N^{2} C\left(q_{i}, q_{j}\right)
$$

Lemma 4.16. The process

$$
\left(\sqrt{n} \frac{\partial}{\partial u} S_{n}(0, q)\right)_{q \in \mathcal{Q}_{\eta}}
$$

is uniformly tight.

Proof of Lemma 4.16. For any $q_{1}, q_{2}$ and any positive integer $k$ let

$$
Z_{k}=\left(\frac{\partial p_{k}}{\partial u}\left(0, q_{1}\right)-\frac{\partial p_{k}}{\partial u}\left(0, q_{2}\right)\right)\left(W_{k}-1\right) .
$$

Using the Rosenthal inequality for martingales (Hall and Heyde [18]) one has for any $p \geq 2$ :

$$
\begin{aligned}
E\left|\sqrt{n}\left(\frac{\partial}{\partial u} S_{n}\left(0, q_{1}\right)-\frac{\partial}{\partial u} S_{n}\left(0, q_{2}\right)\right)\right|^{p} & \leq \mathrm{Cte} \cdot E\left[\left(\frac{1}{n} \sum_{k=1}^{n} E\left(Z_{k}^{2} / Y_{1}, \ldots, Y_{k-1}\right)\right)^{p / 2}\right] \\
& +\frac{1}{n^{p / 2}} \sum_{k=1}^{n} E\left|Z_{k}\right|^{p} .
\end{aligned}
$$

We have now

$$
E\left(Z_{k}^{2} / Y_{1}, \ldots, Y_{k-1}\right)=\left(\frac{\partial p_{k}}{\partial u}\left(0, q_{1}\right)-\frac{\partial p_{k}}{\partial u}\left(0, q_{2}\right)\right)^{2} N^{2}
$$

By iterating equation (15), one obtains for any $q$ :

$$
\frac{\partial p_{k}}{\partial u}(0, q)=q+\sum_{l=1}^{k-2} q(1-q)^{l} W_{k-1} \cdots W_{k-l}+(1-q)^{k-1} W_{k-1} \cdots W_{1}
$$


so that

$$
\left|\frac{\partial p_{k}}{\partial u}\left(0, q_{1}\right)-\frac{\partial p_{k}}{\partial u}\left(0, q_{2}\right)\right| \leq\left|q_{1}-q_{2}\right|\left[1+\sum_{l=1}^{k-1}(1-\tilde{q})^{l-1}|1-(l+1) \tilde{q}| W_{k-1} \cdots W_{k-l}\right]
$$

where $\tilde{q}$ is some number between $q_{1}$ and $q_{2}$.

Define

$$
\alpha=1-\frac{2}{2+\epsilon_{*}} \frac{\log (1-\eta / 4)}{\log \left(1-q_{\eta}\right)} .
$$

We have $0<\alpha<1$, and for big enough $l$ one has

$$
(l+1)\left(1-q_{\eta}\right)^{l-1} \leq\left(1-q_{\eta}\right)^{\alpha l},
$$

so that:

$$
\left|\frac{\partial p_{k}}{\partial u}\left(0, q_{1}\right)-\frac{\partial p_{k}}{\partial u}\left(0, q_{2}\right)\right| \leq \text { Cte }\left|q_{1}-q_{2}\right|\left[1+\sum_{l=1}^{k-1}\left(1-q_{\eta}\right)^{\alpha l} W_{k-1} \cdots W_{k-l}\right] .
$$

Define the Markov chain $\left(D_{k}\right)_{k \in \mathbb{N}}$ by $D_{1}=0$ and

$$
D_{k+1}=1+\left(1-q_{\eta}\right)^{\alpha} W_{k} D_{k} .
$$

Following the arguments of the proof of Lemma 4.14, it can be proved that this Markov chain is Harris positive and $1+x^{2+\epsilon_{*}}$-uniformly ergodic. Now, equation (35) implies that

$$
\left|\frac{\partial p_{k}}{\partial u}\left(0, q_{1}\right)-\frac{\partial p_{k}}{\partial u}\left(0, q_{2}\right)\right| \leq \mathrm{Cte}\left|q_{1}-q_{2}\right| D_{k} .
$$

We thus obtain

$$
\begin{aligned}
E\left[\left(\frac{1}{n} \sum_{k=1}^{n} E\left(Z_{k}^{2} / Y_{1}, \ldots, Y_{k-1}\right)\right)^{p / 2}\right] & \leq \text { Cte }\left|q_{1}-q_{2}\right|^{p} E\left[\left(\frac{1}{n} \sum_{k=1}^{n} D_{k}^{2}\right)^{p / 2}\right] \\
& \leq \text { Cte }\left|q_{1}-q_{2}\right|^{p} E\left[\frac{1}{n} \sum_{k=1}^{n} D_{k}^{p}\right]
\end{aligned}
$$

where the last inequality follows by Jensen's inequality since $p / 2 \geq 1$. But, as soon as $p \leq 2+\epsilon_{*}, \frac{1}{n} \sum_{k=1}^{n} E D_{k}^{p}$ converges and we get:

$$
E\left[\left(\frac{1}{n} \sum_{k=1}^{n} E\left(Z_{k}^{2} / Y_{1}, \ldots, Y_{k-1}\right)\right)^{p / 2}\right] \leq \mathrm{Cte}\left|q_{1}-q_{2}\right|^{p}
$$

for big enough $n$. Also, using (36), as soon as $p \leq 2+\epsilon_{*}$ :

$$
\frac{1}{n^{p / 2}} \sum_{k=1}^{n} E\left|Z_{k}\right|^{p} \leq \mathrm{Cte}\left|q_{1}-q_{2}\right|^{p} \frac{1}{n^{p / 2}} \sum_{k=1}^{n} E D_{k}^{p} \leq \mathrm{Cte}\left|q_{1}-q_{2}\right|^{p}
$$

for big enough $n$. 
It follows from equations $(34,38)$ and $(39)$ that for big enough $n$, for any $q_{1}$ and $q_{2}$,

$$
E\left|\sqrt{n}\left(\frac{\partial}{\partial u} S_{n}\left(0, q_{1}\right)-\frac{\partial}{\partial u} S_{n}\left(0, q_{2}\right)\right)\right|^{2+\epsilon_{*}} \leq \mathrm{Cte} \cdot\left(q_{1}-q_{2}\right)^{2+\epsilon_{*}},
$$

which is the Kolmogorov criterion for the uniform tightness of a continuous process, and Lemma 4.16 follows.

\section{REFERENCES}

[1] L.D. Atwood, A.F. Wilson, J.E. Bailey-Wilson, J.N. Carruth and R.C. Elston, On the distribution of the likelihood ratio test statistic for a mixture of two normal distributions. Comm. Statist. Simulation Comput. 25 (1996) 733-740.

[2] L.E. Baum and T. Petrie, Statistical inference for probabilistic functions of finite state Markov chains. Ann. Math. Stat. 37 (1966) 1554-1563.

[3] P.J. Bickel and Y. Ritov, Inference in hidden Markov models I: Local asymptotic normality in the stationary case. Bernoulli 2 (1996) 199-228.

[4] P.J. Bickel, Y. Ritov and T. Ryden, Asymptotic normality of the maximum-likelihood estimator for general hidden Markov models. Annals of Stat. 26 (1998) 1614-1635.

[5] R.-J. Chuang and N.R. Mendell, The approximate null distribution of the likelihood ratio test for a mixture of two bivariate normal distributions with equal covariance. Comm. Statist. Simulation Comput. 26 (1997) 631-648.

[6] G.A. Churchill, Stochastic models for heterogeneous DNA sequences. Bull. Math. Biology 51 (1989) 79-94.

[7] G. Ciuperca, Sur le test de maximum de vraisemblance pour le mélange de populations. Note aux C.R.A.S., 328, Série I, 4 (1999) 351-358.

[8] D. Dacunha-Castelle and M. Duflo, Probabilités et statistiques, Tome 2. Masson (1993).

[9] D. Dacunha-Castelle and E. Gassiat, Estimation of the number of components in a mixture. Bernoulli 3 (1997a) $279-299$.

[10] D. Dacunha-Castelle and E. Gassiat, Testing in locally conic models. ESAIM Probab. Statist. 1 (1997b).

[11] D. Dacunha-Castelle and E. Gassiat, Testing the order of a model using locally conic parametrization: Population mixtures and stationary ARMA processes. Ann. Statist. 27 (1999) 1178-1209.

[12] A.P. Dempster, N.M. Laird and D.B. Rubin, Large Maximum-likelihood from incomplete data via the EM algorithm. J. Roy. Statist. Soc. Ser. B 39 (1977) 1-38.

[13] R. Douc and C. Matias, Asymptotics of the Maximum Likelihood Estimator for general Hidden Markov Models (1999) (submitted).

[14] M. Duflo, Algorithmes stochastiques. Springer (1996).

[15] Z.D. Feng and C.E. McCulloch, Using bootstrap Likelihood Ratio in Finite Mixture Models. J. Roy. Statist. Soc. Ser. B 58 (1996) 609-617.

[16] L. Finesso, Consistent Estimation of the Order for Markov and Hidden Markov Chains. Ph.D. Thesis, University of Maryland (1990).

[17] D.R. Fredkin and J.A. Rice, Maximum likelihood estimation and identification directly from single-channel recordings. Proc. Roy. Soc. London Ser. B 249 (1992) 125-132.

[18] P. Hall and C.C. Heyde, Martingale Limit Theory and Its Application. Academic Press (1980).

[19] J.A. Hartigan, A failure of likelihood ratio asymptotics for normal mixtures, in Proc. Berkeley Conference in Honor of Jerzy Neyman and Jack Kiefer, edited by L.M. Le Cam and R.A. Olshen (1985) 807-810.

[20] J. Henna, On estimating the number of constituents of a finite mixture of continuous distributions. Ann. Inst. Statist. Math. 37 (1985) 235-240.

[21] J.L. Jensen and N.V. Petersen, Asymptotic normality of the maximum likelihood estimator in state space models. Ann. Statist. 27 (1999) 514-535.

[22] C. Keribin, Tests de modèles par maximum de vraisemblance, Thèse de l'Université d'Evry-Val d'Essonne (1999).

[23] C. Keribin, Consistent estimation of the Order of Mixture Models (1997) (submitted).

[24] B.G. Leroux, Maximum-likelihood estimation for hidden Markov models. Stochastic Process Appl. 40 (1992) 127-143.

[25] B.G. Leroux and M.L. Puterman, Maximum Penalized Likelihood Estimation for Independent and Markov-Dependent Mixture Models. Biometrics 48 (1992) 545-558.

[26] B.G. Lindsay, Mixture models: Theory, Geometry and Applications (1995).

[27] I.L. Mac Donald and W. Zucchini, Hidden Markov and Other Models for Discrete-valued Time Series. Chapman and Hall (1997).

[28] G.J. McLachlan, On Bootstrapping the Likelihood Ratio Test Statistic for the Number of Components in a Normal Mixture. Appl. Statist. 36 (1987) 318-324.

[29] L. Mevel, Statistique asymptotique pour les modèles de Markov cachés. Thèse de l'Université de Rennes I (1997).

[30] L. Mevel and F. LeGland, Exponential forgetting and Geometric Ergodicity in Hidden Markov models. Math. Control Signals Systems (to appear). 
[31] S.P. Meyn and R.L. Tweedie, Markov chains and stochastic stability. Springer-Verlag (1993).

[32] L.R. Rabiner, A tutorial on hidden Markov models and selected applications in speech recognition. Proc. IEEE 77 (1989) $257-284$.

[33] T. Ryden, Estimating the order of hidden Markov models. Statistics 26 (1995) 345-354.

[34] P. Vandekerkhove, Identification de l'ordre des processus ARMA stables. Contribution à l'étude statistique des chaînes de Markov cachées. Thèse de l'Université de Montpellier II (1997).

[35] A. Van der Vaart, Asymptotic Statistics. Cambridge Ed. (1999). 